\title{
High-Temperature Discrete Dislocation Plasticity
}

\author{
S. M. Keralavarma ${ }^{1 *}$ and A. A. Benzerga ${ }^{1,2}$ \\ 1 Department of Aerospace Engineering, Texas A\&M University, \\ College Station, TX 77843 \\ 2 Department of Materials Science $\&$ Engineering, Texas A\&M University, \\ College Station, TX 77843
}

February 19, 2015

\begin{abstract}
A framework for solving problems of dislocation-mediated plasticity coupled with point-defect diffusion is presented. The dislocations are modeled as line singularities embedded in a linear elastic medium while the point defects are represented by a concentration field as in continuum diffusion theory. Plastic flow arises due to the collective motion of a large number of dislocations. Both conservative (glide) and nonconservative (diffusion-mediated climb) motions are accounted for. Time scale separation is contingent upon the existence of quasi-equilibrium dislocation configurations. A variational principle is used to derive the coupled governing equations for point-defect diffusion and dislocation climb. Superposition is used to obtain the mechanical fields in terms of the infinite-medium discrete dislocation fields and an image field that enforces the boundary conditions while the point-defect concentration is obtained by solving the stress-dependent diffusion equations on the same finite-element grid. Core-level boundary conditions for the concentration field are avoided by invoking an approximate, yet robust kinetic law. Aspects of the formulation are general but its implementation in a simple plane strain model enables the modeling of high-temperature phenomena such as creep, recovery and relaxation in crystalline materials. With emphasis laid on lattice vacancies, the creep response of planar single crystals in simple tension emerges as a natural outcome in the simulations. A large number of boundary-value problem solutions are obtained which depict transitions from diffusional to power-law creep, in keeping with long-standing phenomenological theories of creep. In addition, some unique experimental aspects of creep in small scale specimens are also reproduced in the simulations.
\end{abstract}

*Presently at Indian Institute of Technology Madras, Chennai, India 


\section{Introduction}

The objective of this work is to expand the range of application of current discrete dislocation dynamics simulations of crystal plasticity to high homologous temperatures and thus enable mesoscopic simulations of creep and other thermally activated phenomena. The underlying fundamental processes are as relevant to metal plasticity (McDowell, 2008) as they are in geophysics (Castelnau et al., 1996). Plastic deformation of crystalline matter occurs across multiple length and time scales. A discrete dislocation framework based on continuum elasticity theory, e.g. (Van der Giessen and Needleman, 1995; Zbib et al., 1998; Ghoniem et al., 2000; Vattré et al., 2014), constitutes the hyphen between atomicscale descriptions (Bulatov et al., 1998; Shenoy et al., 1999; Shilkrot et al., 2004) and a multitude of meso-scale (El-Azab, 2000; Ispánovity et al., 2014) or macroscopic descriptions of plasticity (Asaro and Needleman, 1985; Fleck and Hutchinson, 1997; Acharya and Bassani, 2000; Gurtin, 2002; Hochrainer et al., 2014). In the sought framework, the continuum theories of elasticity and stress-affected diffusion are used as a basis, and both glide and climb motion of dislocations are accounted for. Climb-enabled dislocation plasticity has known increasing interest in the past few years (Mordehai et al., 2008; Keralavarma et al., 2012; Davoudi et al., 2012; Ayas et al., 2014; Po and Ghoniem, 2014; Geslin et al., 2014); also see Raabe (1998); Gao et al. (2010); Arsenlis et al. (2012) and Geers et al. (2014). This paper contains the theoretical foundations of the creep simulations reported by Keralavarma (2011) and Keralavarma et al. (2012) and reports additional simulations. The key departure from other works is the prescription to simulate creep deformation as an emergent behavior. One major challenge is how to bridge the widely disparate time scales for dislocation glide $(\sim n s$ or smaller $)$ and climb $(\sim \mathrm{ms}$ or larger $)$. Hence, any high-temperature discrete dislocation framework must delineate the conditions under which time-scale separation is possible and implement a computational strategy enabling large-scale simulations.

The microscopic mechanisms of inelastic deformation in crystalline materials depend on the applied stress as well as temperature. Figure 1 illustrates a deformation mechanism map typical of metals. Contours of constant strain rate $\dot{\gamma}$ are plotted in a diagram of shear stress $\tau$ (labeled $\sigma_{s}$ in the figure) normalized by the shear modulus $\mu$ versus the homologous temperature, with $T_{\mathrm{m}}$ denoting the melting point. Such maps are constructed by extensive tabulation of experimental creep data and using phenomenological rate equations of the form

$$
\dot{\gamma} \propto \tau^{n} \bar{d}^{-p}
$$

to interpolate between them (Ashby, 1972; Frost and Ashby, 1982). In (1) $\bar{d}$ refers to the average grain size. The exponents $n$ and $p$ and the temperature-dependent constant of proportionality are determined by a combination of assumptions regarding the microscopic mechanisms and fitting to experimental data. The diagram is divided into regions where the different creep mechanisms predominate. The boundaries between the different regions are determined approximately as the loci of points where competing modes of creep yield equal strain rates according to (1). Plastic flow at low homologous temperatures $\left(T / T_{\mathrm{m}} \sim\right.$ 0.1) essentially arises from the glide of dislocations, which requires stresses about the yield point. In this regime, an increase in dislocation density is usually indicative of an 


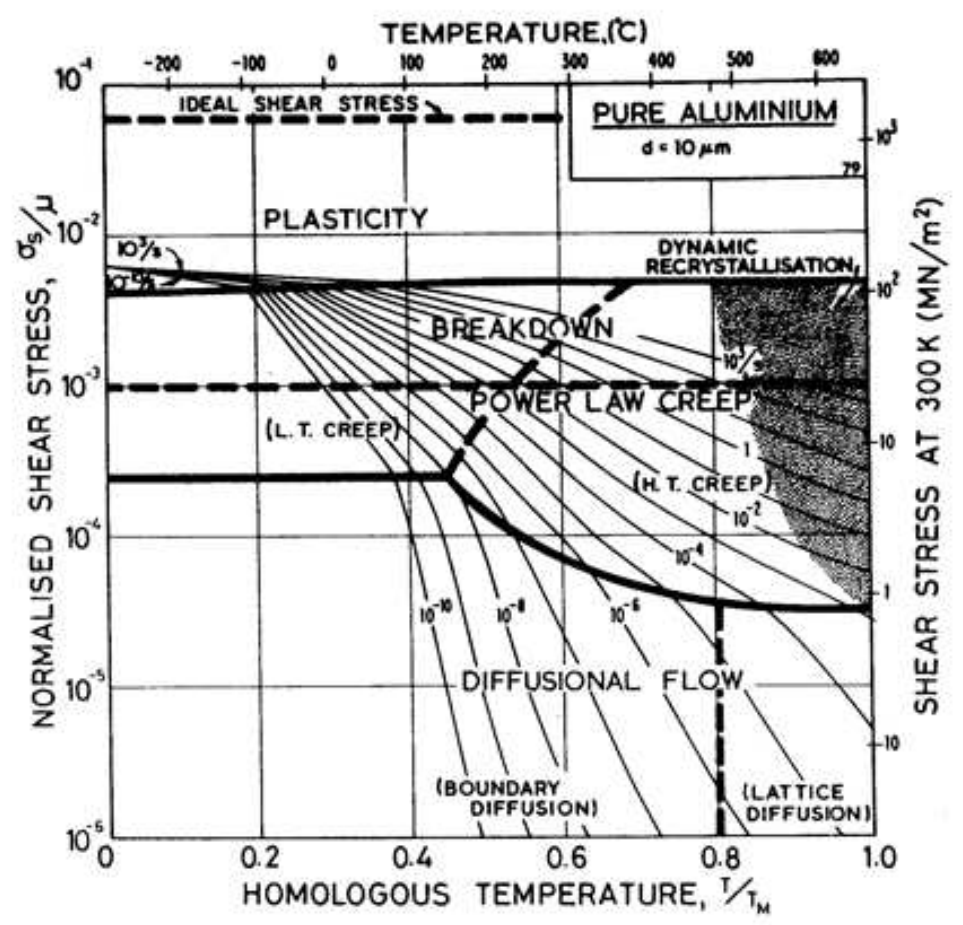

Figure 1: Deformation mechanism map for polycrystalline $\mathrm{Al}$ adapted from Frost and Ashby (1982).

increase in flow stress, for instance due to Taylor hardening. This region corresponds to "plasticity" in Fig. 1. On the other hand, at sufficiently high temperatures, say $T / T_{\mathrm{m}}>$ 0.4 , dislocations can bypass obstacles by several thermally activated mechanisms such as cross-slip and climb. Dislocation climb, aided by the diffusion of vacancies into the dislocation cores, is an important microscopic mechanism for creep and recovery and may control the rate of deformation under some circumstances (Dorn, 1955; Weertman, 1957; Caillard and Martin, 2003). In this regime, materials deform at loads well below their yield stress and their deformation is sustained with no associated increase in stress or dislocation density.

Creep is a thermally activated mode of inelastic deformation whose rate depends on several competing microscopic deformation mechanisms. Part of the strain rate results from mass transport through the diffusive flow of vacancies across a chemical potential gradient due to the imposed loads and interaction with dislocations. This mode of deformation, known as Nabarro-Herring creep (Herring, 1950; Nabarro et al., 1964), is dominant at very low stresses in single crystals or relatively large grain polycrystals and is characterized by exponents $1 \leq n<2$ and $p=2$. Another mode of diffusional creep in polycrystals, known as Coble creep (Coble, 1963), is due to the diffusion of vacancies along grain boundaries. It dominates when lattice diffusion is negligible $\left(0.4<T / T_{\mathrm{m}}<0.8\right)$ with characteristic exponents $1 \leq n<2$ and $p=3$. At higher stresses dislocations play an increasingly important role in creep as they bypass obstacles by climbing normal to their slip planes through absorption or emission of vacancies. This defines the regime of so-called "power-law creep" (Fig. 1) with a stress exponent $n \geq 5$. This regime has itself 
two parts. At high temperatures the bulk concentration of vacancies is sufficient to aid dislocation climb while at lower temperatures the dislocation cores can act as conduits for rapid diffusion of vacancies.

Thus, in terms of deformation mechanism maps, the objective of this work is to extend the range of current discrete dislocation frameworks from the "plasticity" domain down to the "creep" domains in Fig. 1 laying emphasis on single crystals and leaving out dislocation-core processes such as pipe diffusion. The significance of undertaking this task is two-fold. First, it enables fundamental understanding of temperature-dependent plastic/creep deformation as an emergent behavior. This entails obtaining constitutive relations from initial/boundary-value problem solutions, including strain partitioning, evolution of the dislocation structure and the elastic energy stored therein, as was done for example for low-temperature deformation (Benzerga et al., 2004, 2005). Second, a framework that captures the salient features of bulk plasticity provides a sound basis for understanding creep in small-scale systems or ultra-fine grained and nanocrystalline materials for which deviations from bulk behavior begin to be documented ( $\mathrm{Ng}$ and Ngan, 2007; Meyers et al., 2006).

Previous attempts at incorporating dislocation climb in dislocation dynamics (DD) simulations began with glide-like viscous drag models (Xiang et al., 2004; Hartmaier et al., 2005) which ignore the effects of vacancy diffusion on climb motion altogether. Diffusion of vacancies and dislocation climb are coupled phenomena in the sense that climb is a non-conservative motion that leads to the emission or absorption of point defects. The diffusion of the latter under imposed pressure and concentration gradients leads to an "osmotic" force on the dislocations normal to their slip planes. Thus, at high temperatures and/or high vacancy supersaturations, dislocations may climb under the effect of this osmotic force, even in the absence of a mechanical (Peach-Koehler) force. Mordehai et al. (2008) developed an analytical climb kinetic model that takes explicit account of the osmotic force contribution using equilibrium solutions for the climb rate of dislocations in a prescribed uniform vacancy field. Their kinetic law was used in 3D DD simulations of diffusion-controlled dislocation loop coarsening (Bakó et al., 2011) and irradiation hardening in BCC iron (Arsenlis et al., 2012) as well as in 2D DD simulations of thin films (Davoudi et al., 2012) and creep (Keralavarma et al., 2012). Gao et al. (2010) included the effect of pipe diffusion in a 3D DD simulation, but neglecting bulk diffusion. More recently, Geslin et al. (2014) formulated a phase-field model of dislocation climb which accounts for both bulk diffusion and emission/absorption kinetics at the core level. They also proposed an extension of the kinetic law of Mordehai et al. (2008) which allows to account for pipe diffusion and jog density; also see Li et al. (2012) for an earlier attempt using the phase field method. Only recently have discrete dislocation formulations that include climb and account for relevant thermodynamic constraints been undertaken (Gao and Cocks, 2009; Po and Ghoniem, 2014). Ayas et al. (2014) developed a methodology for diffusion mediated dislocation climb where the diffusive flux of vacancies is computed using a superposition scheme similar to that for the elastic fields. They looked at the effect of enabling dislocation climb on plasticity under tensile and bending boundary conditions. Unlike their approach, where an artificially large value for the diffusion coefficient was used to promote dislocation climb under the simulated time scales, we adopt an adaptive, 
staggered time stepping approach in order to tackle creep problems over much longer time scales.

The formulation is applicable in three dimensions. However, the complexity involved makes large scale creep simulations out of reach. The detailed formulation of the coupled boundary value problems of dislocation dynamics and vacancy diffusion is described in Section 2. This extends the discrete dislocation plasticity framework of Van der Giessen and Needleman (1995) to higher temperatures and the thermodynamic variational framework of Gao and Cocks (2009) to coupled dislocation-glide and climb for a multi-dislocation system in a finite body. Evolution equations are derived for the microstructural variables, namely the positions of the discrete dislocations and the vacancy concentration field, and constitutive rules are framed for the climb of dislocations as a result of interactions with the vacancy cloud. Section 3 describes the solution methodology for the creep boundary value problem and implements time scale separation. Simulation results are presented therein and discussed in a broader context in the last section.

\section{Formulation}

Consider a body occupying domain $V$ containing a set of $N$ discrete edge dislocations and a dilute concentration of diffusing species, as sketched in Fig. 2. The position of dislocation $i$ at time $t$ is denoted $\mathbf{x}^{i}(t)$. The core region of a dislocation $i$ is denoted $C^{i}$, their union

$$
\tilde{C}=\bigcup_{i=1}^{N} C^{i},
$$

and $\hat{V}=V \backslash \tilde{C}$ denotes the volume of the body excluding all dislocation cores. The body is subjected to mixed traction/displacement boundary conditions, Fig. 2a. Let $c(\mathbf{x}, t)$ denote the fractional concentration, with $\mathbf{x}$ denoting the position and $t$ the time. It is defined as the ratio of the number of diffusing species to the number of lattice sites in an elementary volume. It is assumed that any species that enters the core region of a dislocation is immediately absorbed by the dislocation so that $c(\mathbf{x}, t) \equiv 0$ in $\tilde{C}$.

\subsection{Time scale separation}

Dislocation glide and climb are kinetic processes that occur over widely differing time scales. Comparison of the glide and climb velocities using basic analytical estimates (Hirth and Lothe, 1968) shows that dislocation glide velocities exceed the climb velocity by several orders of magnitude except at temperatures very close to the melting point. Therefore, discrete dislocation simulations of a slow process such as creep present a challenge in terms of resolving both glide and climb events within realistic computation times. We address this issue by postulating the existence of 'quasi-equilibrium' states in a dynamical system consisting of a large number of glide dislocations. The postulate is based on the observation from DD simulations that, at constant applied stresses below the nominal yield stress and in the absence of climb, the average strain attains a constant value in a relatively short span of time compared with the time scale of the climb process. Two 

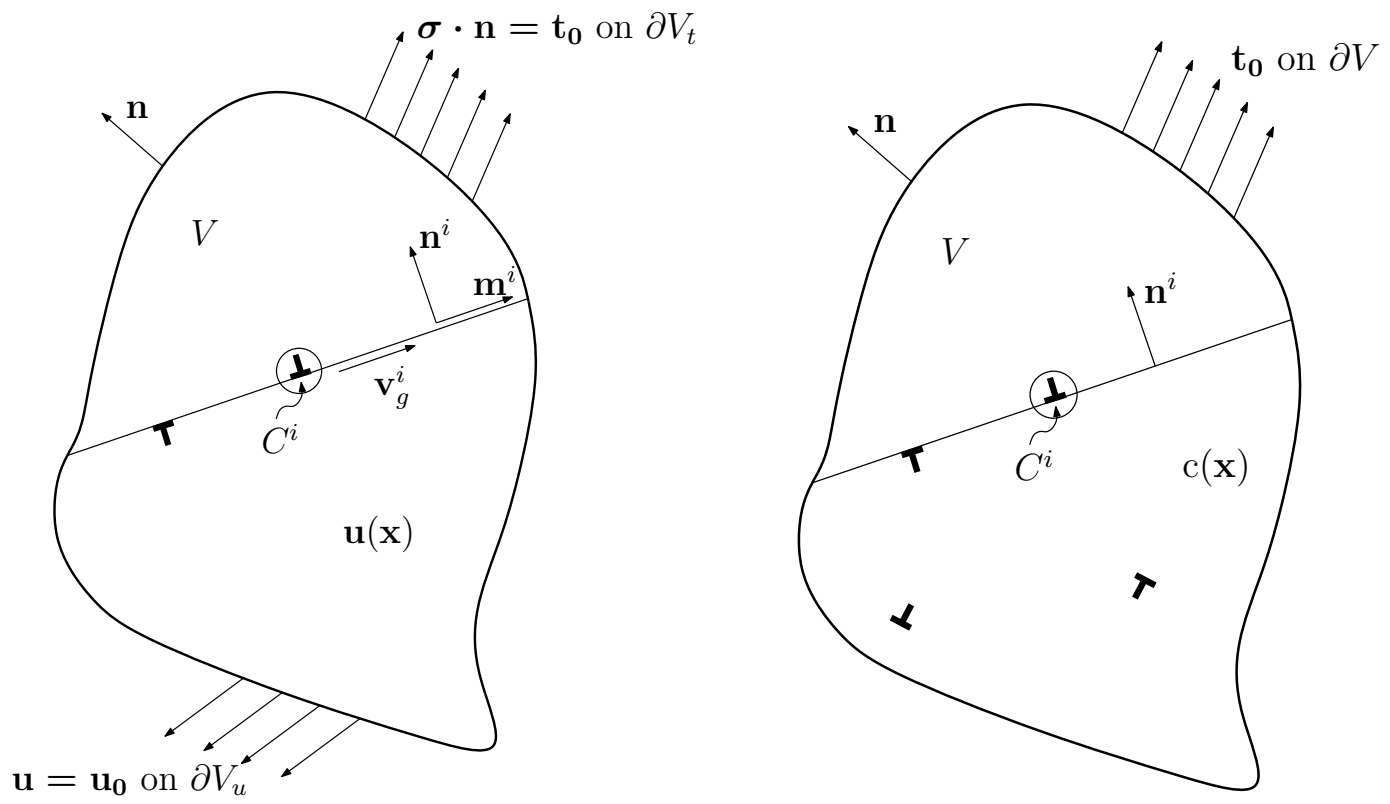

Figure 2: Sequential solution of (a) the linear elasticity and (b) unsteady diffusion boundary value problems with time steps $t_{\mathrm{gl}}$ in (a) and $t_{\mathrm{cl}}(T)$ in (b) given that $t_{\mathrm{cl}} \gg t_{\mathrm{gl}}$.

consequences follow. First, since the quasi-equilibrium states are characterized by reduced glide activity, one can focus on climb activity freezing dislocation glide altogether. It is emphasized that, microscopically, glide activity never completely ceases in such a system. Second, because such states are attained 'quickly' dislocation climb may be effectively neglected during the short, glide-dominated interval. Under such circumstances, individual dislocation climb motions are the 'activation' events that enable the entire system to move from one quasi-equilibrium state to the next. The above postulate is consistent, as are its consequences, with views of creep as a result of the thermally activated climb of dislocations from local equilibrium positions (Hirth and Lothe, 1968; Caillard and Martin, 2003).

In practice, therefore, we model the overall deformation as a sequence of glide and climb steps rather than a continuous process of simultaneously occurring glide and climb. In this framework, the glide steps (Fig. 2a) are performed using the discrete dislocation formulation of Van der Giessen and Needleman with minor changes to account for the effect of temperature on some of the constitutive rules, described in section 2.5. The climb steps (Fig. 2b) are computed using a coupled formulation involving point-defect diffusion and dislocation climb described below.

The general formulation is applicable in 3D and to any diffusing species responsible for dislocation climb. However, the physics of point-defect dislocation interactions may vary. Below, we focus on lattice vacancies as the only diffusing species. 


\subsection{Climb Problem Formulation}

In this section, we focus on the unsteady diffusion boundary value problem (Fig. 2b) during a climb time step $t_{\mathrm{cl}}(T)$ with no glide activity. We derive the governing equations for coupled vacancy diffusion and dislocation climb. The body is subjected on its boundary $\partial V$ to tractions $\mathbf{t}^{0}$, viewed as those obtained from the solution of the elastic boundary value problem (Fig. 2a).

\subsubsection{Thermodynamic potentials}

The response of the body is fully determined by the specification of two thermodynamic potentials: the Gibbs free energy, $G$, and the dissipation, $\Psi$. The former is written as

$$
G=G\left(c(\mathbf{x}, t), \mathbf{x}^{i}(t)\right)=\int_{V} g \mathrm{~d} V
$$

with $g(\mathbf{x}, t)=h-T s$ the Gibbs free energy density in $V$ and $h$ and $s$ are the enthalpy and entropy per unit volume, respectively. Both $h$ and $s$ depend on the elastic and vacancy fields and are given by

$$
h=\frac{1}{2} \boldsymbol{\sigma}: \boldsymbol{\epsilon}+\left[\frac{E_{f}}{\Omega}-p \frac{\Omega_{v}}{\Omega}\right] c-\boldsymbol{\sigma}:\left(\boldsymbol{\epsilon}+\boldsymbol{\epsilon}^{v}\right)
$$

and

$$
s=-\frac{k}{\Omega}[c \log c+(1-c) \log (1-c)]
$$

see, e.g. (Cottrell, 1953). Above, $\boldsymbol{\sigma}$ denotes the stress tensor, $\boldsymbol{\epsilon}$ is the lattice strain, $\boldsymbol{\epsilon}^{v}$ is an inelastic strain due to the diffusion of vacancies, $p=-\operatorname{tr}(\boldsymbol{\sigma}) / 3$ is the hydrostatic pressure field, $E_{f}$ is the formation energy of a single vacancy (energy required to break the atomic bonds), $\Omega$ is the atomic volume, $\Omega_{v}$ is the relaxation volume of a vacancy and $k$ is the Boltzmann constant. The relaxation volume $\Omega_{v}$ is the reduction in crystal volume as a result of the removal of an atom and is typically a fraction of the atomic volume. Contributions to the lattice strain $\boldsymbol{\epsilon}$ due to vacancies are excluded because they are short-ranged and the associated strain energy is of second order in the concentration $c$ (Garikipati et al., 2006). On the other hand, the interaction energy between the external field and the vacancy strain fields scales with $c$. For a homogeneous external field, this energy is to first order the work done by the hydrostatic stress, $-p$, against the reduction in crystal volume (Cottrell and Jaswon, 1949; Bullough and Newman, 1970), which is the origin of the third term in (3). Inside the cores $\tilde{C}$, all the terms in $g$ involving the vacancy concentration $c$ vanish identically due to the assumption that $c \equiv 0$ therein. The expression for the total Gibbs free energy follows by substituting (3)-(4) in (2), which is then decomposed into two terms:

$$
G=G_{1}\left(\mathbf{x}^{i}(t)\right)+G_{2}\left(c(\mathbf{x}, t), p\left(\mathbf{x}^{i}(t)\right)\right) .
$$

Here, $G_{1}$ denotes the contribution from the elastic strain energy

$$
G_{1}=\int_{\hat{V}} \frac{1}{2} \boldsymbol{\sigma}: \boldsymbol{\epsilon} \mathrm{d} V+\sum_{i=1}^{N} \int_{\partial C^{i}} \frac{1}{2} \mathbf{t} \cdot \mathbf{u} \mathrm{d} S-\int_{\partial V} \mathbf{t}_{0} \cdot \mathbf{u} \mathrm{d} S
$$


and $G_{2}$ denotes the contribution from the thermally generated vacancies

$$
G_{2}=\int_{\hat{V}}\left\{\left[\frac{E_{f}}{\Omega}-p \frac{\Omega_{v}}{\Omega}\right] c+\frac{k T}{\Omega}[c \log c+(1-c) \log (1-c)]\right\} \mathrm{d} V-\int_{\partial V}\left(\mathbf{t}_{0} \cdot \mathbf{n}\right) u^{v} \mathrm{~d} S
$$

where $\mathbf{t}$ is the traction, $\mathbf{u}$ the displacement due to elasticity alone, and $u^{v} \mathbf{n}$ denotes the motion of the boundary normal to itself due to the diffusive flux of mass though it. The surface integrals over $\partial C^{i}$ and $\partial V$ are obtained by application of the divergence theorem, following the procedure of Van der Giessen and Needleman (1995), with due consideration given to the singularity of the elastic strain energy in $\tilde{C}$. $G_{1}$ is a purely mechanical contribution to the free energy due to the elastic fields while $G_{2}$ represents the contribution from the vacancies and is coupled to the elastic fields through the hydrostatic pressure $p$.

During the climb time steps, energy dissipation in the system is entirely due to the diffusive flux of the vacancies, for which a quadratic form for the dissipation potential $\Psi$ is assumed following Gao and Cocks (2009)

$$
\Psi=\frac{1}{2} \int_{\hat{V}} \frac{1}{\bar{D}} \mathbf{j} \cdot \mathbf{j} \mathrm{d} V
$$

where $\mathbf{j}$ is the volumetric flux (in $\mathrm{m} / \mathrm{s}$ ) and $\bar{D}=D \Omega c(1-c) / k T$. The vacancy diffusion coefficient $D$ in solids depends strongly on temperature and weakly on pressure. Here, we neglect the pressure dependence of $D$, so that a uniform value of $D$, independent of the dislocation positions, is used. An Arrhenius type equation is used to express the temperature dependence of $D$ :

$$
D=D_{0} \exp \left(-\frac{E_{m}}{k T}\right)
$$

where $E_{m}$ is the vacancy migration energy and $D_{0}$ is the asymptotic value of the vacancy diffusion coefficient at very high temperatures; see (Hirth and Lothe, 1968).

\subsubsection{Driving forces}

The thermodynamic driving force for self-diffusion is the gradient of the chemical potential of the vacancies. The latter depends on the dislocation positions, since climbing dislocations can act as sources/sinks for vacancies and the hydrostatic component of the dislocation stress field modifies the local chemical potential.

The rate of change of the Gibbs free energy is obtained from (5) as:

$$
\dot{G}=-\sum_{i=1}^{N} \mathbf{f}^{i} \cdot \mathbf{v}^{i}+\int_{\hat{V}}\left(\mu_{v} \dot{c}-\frac{\Omega_{v}}{\Omega} c \dot{p}\right) \mathrm{d} V+\int_{\partial V}\left(\mathbf{t}^{0} \cdot \mathbf{n}\right)(\mathbf{j} \cdot \mathbf{n}) \mathrm{d} S
$$

where $\mathbf{f}^{i}$ denotes the Peach-Koehler force on dislocation $i, \mathbf{v}^{i}$ its velocity, $\mu_{v} \equiv \partial g / \partial c$ denotes the chemical potential of the vacancies, and $\mathbf{j}$ as above. Mass conservation was 
used in the form $\dot{u}^{v}=-\mathbf{j} \cdot \mathbf{n}$ on the external boundary $\partial V$. The expression for the PeachKoehler force $\mathbf{f}^{i}$ in a body containing multiple dislocations has been derived by Van der Giessen and Needleman (1995) and reads

$$
\mathbf{f}^{i}=-\frac{\partial G_{1}}{\partial \mathbf{x}^{i}}=\mathbf{t}^{i} \times\left[\left(\hat{\boldsymbol{\sigma}}+\sum_{j \neq i} \boldsymbol{\sigma}^{j}\right) \cdot \mathbf{b}^{i}\right]
$$

where $\mathbf{t}^{i}$ is a unit vector tangent to the dislocation line and $\mathbf{b}^{i}$ is the Burgers vector. Also, $\boldsymbol{\sigma}^{j}$ is the infinite medium stress field of dislocation $j$ and $\hat{\boldsymbol{\sigma}}$ is the image stress computed by solving the boundary-value problem depicted in Fig. 2a. The chemical potential for the vacancies $\mu_{v}$ is obtained constitutively from equations (3)-(4) as

$$
\mu_{v}=\frac{\partial g}{\partial c}=\frac{k T}{\Omega}\left[\frac{E_{f}}{k T}-\frac{p \Omega_{v}}{k T}+\log \frac{c}{(1-c)}\right]
$$

Ignoring the elastic fields of the vacancies themselves, $p$ is a function of the positions of the dislocations $\mathbf{x}^{i}(t)$. Since $p=p\left(\mathbf{x}^{i}(t)\right)$, one may write

$$
\dot{p}=\sum_{i=1}^{N} \frac{\partial p}{\partial \mathbf{x}^{i}} \cdot \mathbf{v}^{i}
$$

Substituting (13) in (10) and regrouping yields

$$
\dot{G}=\int_{\hat{V}} \mu_{v} \dot{c} \mathrm{~d} V+\int_{\partial V}\left(\mathbf{t}^{0} \cdot \mathbf{n}\right)(\mathbf{j} \cdot \mathbf{n}) \mathrm{d} S-\sum_{i=1}^{N}\left(\mathbf{f}_{d}^{i}+\mathbf{f}^{i}\right) \cdot \mathbf{v}^{i}
$$

where

$$
\mathbf{f}_{d}^{i} \equiv \int_{\hat{V}} c \frac{\Omega_{v}}{\Omega} \frac{\partial p}{\partial \mathbf{x}^{i}} \mathrm{~d} V
$$

is a drag force arising from the interaction of the moving dislocations with the vacancy field. This force appears because of the dependence of the chemical potential on the pressure field $p$ so that changes in the latter due to dislocation motion give rise to a thermodynamic force acting on the dislocation.

Mass conservation dictates that the diffusion flux $\mathbf{j}$ and the rate of change of vacancy concentration $\dot{c}$ are related by the continuity equation:

$$
\dot{c}=-\nabla \cdot \mathbf{j} \quad \text { in } \hat{V}
$$

Substituting back in (14) and using the divergence theorem, we get

$$
\dot{G}=\int_{\hat{V}} \nabla \mu_{v} \cdot \mathbf{j} \mathrm{d} V-\int_{\partial V} \mu_{v} \mathbf{j} \cdot \mathbf{n} \mathrm{d} S-\sum_{i=1}^{N} \int_{\partial C^{i}} \mu_{v} \mathbf{j} \cdot \mathbf{n} \mathrm{d} S+\int_{\partial V}\left(\mathbf{t}^{0} \cdot \mathbf{n}\right)(\mathbf{j} \cdot \mathbf{n}) \mathrm{d} S-\sum_{i=1}^{N}\left(\mathbf{f}_{d}^{i}+\mathbf{f}^{i}\right) \cdot \mathbf{v}^{i}
$$

Further, since pure climb motion is assumed for the problem in Fig. $2 \mathrm{~b}$ we have $\mathbf{v}^{i}=v_{c}^{i} \mathbf{n}^{i}$, where $\mathbf{n}^{i}$ denotes the normal to the slip plane of dislocation $i$. In such case, conservation 
of mass can also be invoked to relate the dislocation climb velocity $v_{c}^{i}$ to the flux of vacancies into or away from the dislocation core, since climb must be accompanied by the production/absorption of vacancies at the dislocation core. We obtain the climb velocity from the mass conservation condition as

$$
v_{c}^{i}=\frac{1}{b^{i}} \oint_{\Gamma^{i}} \mathbf{j} \cdot \mathbf{n} \mathrm{d} s=\frac{1}{b^{i} \mathcal{L}^{i}} \int_{\partial C^{i}} \mathbf{j} \cdot \mathbf{n} \mathrm{d} S
$$

where $b^{i}$ is the magnitude of the Burgers vector for dislocation $i, \Gamma^{i}$ the transverse trace of $\partial C^{i}, \mathcal{L}^{i}$ is the length of the dislocation loop and the unit normal $\mathbf{n}$ points into the dislocation core. Note that the second equality in (17) yields the average climb velocity of a dislocation loop as opposed to the local climb velocity given by $(17)_{1}$. The above integral

must be independent of the size of the dislocation core $C^{i}$ by the continuity condition since dislocations are the only sources or sinks of vacancies in the body. Substituting $(17)_{2}$ in (16) we finally get

$$
\dot{G}=\int_{\hat{V}} \nabla \mu_{v} \cdot \mathbf{j} \mathrm{d} V-\int_{\partial V}\left(\mu_{v}-\mathbf{t}^{0} \cdot \mathbf{n}\right) \mathbf{j} \cdot \mathbf{n} \mathrm{d} S+\sum_{i=1}^{N} \frac{1}{b^{i}}\left(f_{o}^{i}+f_{d c}^{i}+f_{c}^{i}\right) \int_{\partial C^{i}} \mathbf{j} \cdot \mathbf{n} \mathrm{d} S
$$

where $f_{c}^{i}$ and $f_{d c}^{i}$ denote the climb components of the Peach-Koehler force and the drag force per unit length of the dislocation respectively, and $f_{o}^{i} \equiv \mu_{v} b^{i}$ is the so called osmotic force per unit length in the climb direction. Physically, (18) embodies the fact that in the absence of glide, the rate of change of the Gibbs free energy is solely due to the diffusive flux of vacancies, as driven by gradients in the chemical potential (first term), applied tractions on the exterior boundary (second term) and three thermodynamic forces acting on the discrete dislocations (last term). One of these forces, $f_{c}^{i}$, is clearly configurational: it is the climb component of the Peach-Koehler force. The osmotic force $f_{o}^{i}$ is purely chemical and may be of the same order of magnitude as $f_{c}^{i}$, as will be illustrated below. Finally, the drag force $f_{d c}^{i}$ results from the interaction of the moving dislocations with the vacancy field due to the pressure-dependence of the chemical potential.

\subsubsection{Variational principle}

Cocks and co-workers (Cocks, 1996; Gao and Cocks, 2009) have shown that the governing equations for self-diffusion may be derived from the stationary value of the following functional

$$
\Pi=\dot{G}+\Psi
$$

Using (18) and (8) in (19) and taking the first variation of $\Pi$ with respect to the vacancy flux yields

$$
\delta \Pi=\int_{\hat{V}}\left(\nabla \mu_{v}+\frac{\mathbf{j}}{\bar{D}}\right) \cdot \delta \mathbf{j} \mathrm{d} V+\int_{\partial V}\left(\mathbf{t}^{0} \cdot \mathbf{n}-\mu_{v}\right)(\delta \mathbf{j} \cdot \mathbf{n}) \mathrm{d} S-\sum_{i=1}^{N} \frac{1}{b^{i}}\left(f_{o}^{i}+f_{d c}^{i}+f_{c}^{i}\right) \int_{\partial C^{i}} \delta \mathbf{j} \cdot \mathbf{n} \mathrm{d} S
$$


From a stationary $\Pi$ with respect to $\mathbf{j}$, one can write the local form of the governing equations for vacancy diffusion as

$$
\begin{array}{rr}
\mathbf{j}=-\bar{D} \nabla \mu_{v} & \text { in } \hat{V} \\
\mu_{v}=\mathbf{t}^{0} \cdot \mathbf{n} & \text { on } \partial V \\
\mu_{v}=-\frac{1}{b^{i}}\left(f_{d c}^{i}+f_{c}^{i}\right) & \text { on } \partial C^{i}
\end{array}
$$

Equation (21) is the constitutive law for vacancy diffusion, which reduces to Fick's first law in the absence of pressure gradients, i.e. $\mathbf{j}=-D \nabla c$. Together with the continuity equation (15) it defines the field equation governing $\mathbf{j}$ or $c$. Equations (22) and (23) are natural boundary conditions that follow from the application of the variational principle. Condition (22) is computationally tractable and shows that the chemical potential, hence the concentration field, on the outer boundary is set by specifying the tractions resulting from solving the elasticity boundary value problem in Fig. 2a. On the other hand, conditions (23) are not tractable since their implementation would require a spatial discretization that (i) resolves dislocation cores and (ii) is adaptive. In addition, there are as many conditions (23) as there are dislocations in the body. In what follows, we seek approximations that render the coupled climb-diffusion problem computationally tractable.

\subsection{Kinetics of Climb}

Dislocation climb is coupled to the flux of vacancies into the dislocation cores and hence the dislocation climb velocities $v_{c}^{i}$ are determined using equation (17) in which the diffusion flux is obtained by solving (21)-(23) along with (15). In this process, the dependence of $v_{c}^{i}$ upon the Peach-Koehler and drag forces is implicit through boundary conditions (23) on the dislocation cores. However, the solution of problem $\{(15),(21)-(23)\}$ for a large scale DD simulation presents a major difficulty due to the need to resolve the cores of dislocations $(\mathrm{O}(\sim 1 \mathrm{~nm}))$ in the numerical discretization. Moreover, since the dislocations themselves are mobile, one would need to adaptively re-mesh the domain so as to track the moving core regions. Both requirements make large scale simulations numerically intractable.

In order to overcome this difficulty, we seek an approximate climb kinetic law, as an alternative to implicit law (17). We first assume separation of scales whereby the macroscopic gradients of vacancy concentration have a characteristic length that is at least an order of magnitude larger than the radius of the dislocation cores. By way of consequence, the global vacancy diffusion problem can be tackled using a relatively coarse mesh $(\mathrm{O}(\sim$ $100 \mathrm{~nm})$ ). This, however, amounts to ignoring boundary conditions (23). Therefore, a new kinetic law that specifies the dependence of the climb velocity upon the driving forces is needed. To this end, the climb velocities are determined using an approximate analytical expression derived by Mordehai et al. (2008), generalizing a previous result from the literature (Hirth and Lothe, 1968). They derived an expression for the climb velocity of an edge dislocation in a prescribed uniform remote vacancy field using the simplifying assumptions of (i) steady state conditions and (ii) radial symmetry of the vacancy 
flux (i.e. neglecting the effect of local gradients in the hydrostatic stress field around the dislocation core). Under these conditions a radially symmetric solution to the continuity equation (15) can be derived, from which the dislocation climb velocity follows using (17) as

$$
v_{c}^{i}=-\eta \frac{D}{b^{i}}\left[c_{0} \exp \left(-\frac{f_{c} \Omega}{b^{i} k T}\right)-c\right]
$$

where

$$
c_{0}=\exp \left(-\frac{E_{f}}{k T}\right)
$$

is the equilibrium vacancy concentration at temperature $T$, and $\eta$ is a constant of order unity. The first term in the square brackets of (24) represents the concentration of vacancies in equilibrium with the dislocation core while $c$ denotes the remote concentration, which is obtained from the solution of the global diffusion boundary value problem interpolated to the dislocation position. The mesoscopic kinetic law (24) has proven robust against atomistic simulations and seems necessary to extrapolate them in a range of dislocation densities corresponding to experiments (Clouet, 2011).

Notice that the dislocation climb velocity vanishes when the remote vacancy concentration field equals the equilibrium vacancy concentration around the dislocation core. This condition corresponds to

$$
-f_{c}=\frac{b k T}{\Omega} \log \left(\frac{c_{\infty}}{c_{0}}\right) \approx b \mu_{v}=f_{o}
$$

where the term involving the relaxation volume $\Omega_{v}$ and second and higher order terms in $c$ in the expression for the chemical potential (12) are omitted. Thus, the stationary condition for the climb velocity corresponds to the balance of thermodynamic forces on the dislocation.

\subsection{Updated Climb Problem Formulation}

In the proposed framework, scale separation is assumed and equation (24) for dislocation climb kinetics is employed. Dislocation cores are smeared out and the governing equations for the coarse-grained vacancy concentration field $C$ or the diffusion flux $\mathbf{J}$ are extended to the entire body $V$ instead of $\hat{V}$ for their microscopic counterparts. Consequently, the governing equations for the boundary value problem (15) and (21)-(23) are amended as

$$
\begin{array}{cr}
\dot{C}=-\nabla \cdot \mathbf{J}+\dot{C}_{\mathrm{src}} & \text { in } V \\
\mathbf{J}=-\bar{D} \nabla \mu_{v} & \text { in } V \\
\mu_{v}=\mathbf{t}^{0} \cdot \mathbf{n} & \text { on } \partial V
\end{array}
$$

In (26) the term $\dot{C}_{\mathrm{src}}$ accounts for the production or consumption of vacancies due to dislocation climb of any dislocation located within the elementary volume $V_{g}$ used for coarse-graining. This amendment to the continuity equation is required by mass conservation when the processes of core-level absorption or emission are not actually modeled. 
Under such circumstances, the time rate of change of the source term is estimated from the net climb velocity as (see Appendix):

$$
\dot{C}_{\mathrm{src}}=-\frac{1}{V_{g}} \sum_{i \in V_{g}} \oint_{\mathcal{L}^{i}} b_{e}^{i} v_{c}^{i} d l
$$

where $b_{e}^{i}$ denotes the edge component of dislocation $i$ and $\mathcal{L}^{i}$ the line length within $V_{g}$. In particular, for a collection of parallel straight edge dislocations, one may write

$$
\dot{C}_{\mathrm{src}} V_{g}=-b^{2} \sum_{i \in V_{g}} v_{c}^{i}
$$

As discussed in Appendix, heuristic equation (30) underestimates the source/sink term for a jogged dislocation and alternative heuristics are possible. What is of importance here is to note that the explicit inclusion of $V_{g}$ in (29) entails a length scale $l_{g}$ (with $V_{g} \sim l_{g}^{3}$ ) associated with coarse graining. Consistent with the length scale separation postulate, the length $l_{g}$ is much greater than the dislocation core size (in fact even greater than the dislocation spacing) but sufficiently small compared with specimen dimensions. The source term may thus be considered as a nonlocal term in the global problem. Apart from this amendment to the continuity equation, (27) is the same as (21) but with $V$ replaced with $\hat{V}$ and boundary condition (28) is exactly (22). Boundary conditions (23) are supplanted by amending (26) with the source term $\dot{C}_{\text {src }}$ specified through (29) and the new kinetic law (24).

The modified governing equations for diffusion (26)-(28) do not involve boundary conditions specified on the dislocation core boundaries and hence do not require an atomistically resolved mesh for its solution. Hence, the finite element method and a relatively coarse mesh is used to solve equations (26)-(28). Combining the two equations (26) and (27) and using equation (12) for the chemical potential, one obtains the following nonlinear partial differential equation governing the vacancy concentration field:

$$
\dot{C}=D \nabla^{2} C-\frac{D \Omega_{v}}{k T} \nabla \cdot[C(1-C) \nabla p]+\dot{C}_{\mathrm{src}}
$$

The equation is non-linear in $C$ due to the presence of the term $C(1-C)$. However, since typically $C \ll 1$, (31) is linearized into

$$
\dot{C}=D \nabla^{2} C-\frac{D \Omega_{v}}{k T} \nabla \cdot C \nabla p+\dot{C}_{\mathrm{src}} \quad \text { in } V
$$

This equation is linear since $p$ is a known field, determined from the solution of the elasticity problem. The solution to (32) is obtained subject to Dirichlet boundary conditions for $C$, which may be obtained from (28) combined with (12) to write

$$
C=C_{0} \exp \left(\frac{\mathbf{t}^{0} \cdot \mathbf{n} \Omega}{k T}\right) \quad \text { on } \partial V
$$


where $C_{0}$ is the equilibrium vacancy concentration defined following equation (24), and $\mathbf{t}^{0}$ and $\mathbf{n}$ are the traction and unit normal vectors at the boundary respectively. The weak form for equation (32) may be written as

$$
\int_{V} v \dot{C} \mathrm{~d} V=-D \int_{V} \nabla v \cdot \nabla C \mathrm{~d} V+\frac{D \Omega_{v}}{k T} \int_{V} C \nabla v \cdot \nabla p \mathrm{~d} V+\int_{V} v \dot{C}_{\text {src }} \mathrm{d} V
$$

where $v \in \mathcal{V}$ is an arbitrary test function belonging to the function space

$$
\mathcal{V}=\{w: V \rightarrow \mathbb{R}, \quad w=0 \text { on } \partial V\}
$$

Bilinear quadrilateral elements are used in the finite element discretization of the above problem and the resulting system of ordinary differential equations are integrated in time using an implicit sparse solver from the ODEPACK open source library (Hindmarsh et al., 1983).

\subsection{Governing Equations for Dislocation Glide}

The formulation of the dislocation glide problem (Fig. 2a) is essentially unchanged from the original model of Van der Giessen and Needleman (1995), whose key elements are briefly recalled here. Glide results from the component of the Peach-Koehler force (11) along the slip direction, $f_{g}^{i}$, given by

$$
f_{g}^{i}=\mathbf{n}^{i} \cdot\left[\hat{\boldsymbol{\sigma}}+\sum_{j \neq i} \boldsymbol{\sigma}^{j}\right] \cdot \mathbf{b}^{i}
$$

where $\mathbf{n}^{i}$ is the slip plane normal and other quantities have been defined after (11). The calculation of this force requires knowledge of the elastic fields in the body. The superposition method of linear elasticity is invoked to write

$$
\boldsymbol{\sigma}=\tilde{\boldsymbol{\sigma}}+\hat{\boldsymbol{\sigma}}, \quad \boldsymbol{\epsilon}=\tilde{\boldsymbol{\epsilon}}+\hat{\boldsymbol{\epsilon}}, \quad \mathbf{u}=\tilde{\mathbf{u}}+\hat{\mathbf{u}}
$$

where $\boldsymbol{\sigma}, \boldsymbol{\epsilon}$ and $\mathbf{u}$ denote the total stresses, strains and displacements, respectively. The fields with overscript $(\sim)$ denote the singular elastic fields of the dislocations in an infinite medium and the $\left({ }^{\wedge}\right)$ fields are smooth complementary fields that enforce the boundary conditions for the problem. The ( ) fields are known analytically for infinitely long straight edge dislocations while the $\left({ }^{\wedge}\right)$ fields are computed numerically.

A subtle issue that relates to the definition of the $(\sim)$ fields in a combined glide/climb formulation in two dimensions is the treatment of displacement discontinuities in the specimen as the dislocation structure evolves. A dislocation dipole on a slip plane will have a slip discontinuity equal in magnitude to the Burgers vector extending between them. When one of the two dislocations climbs the earlier discontinuity still terminates at the original position of the dislocation that climbed, while a new discontinuity extends along the climb path as though there is interpenetration of matter over a width equal in magnitude to the Burgers vector. The result is an incompatible displacement field corresponding to the sum of the eigenstrains of two non-existent opposite signed dislocations 
(a)

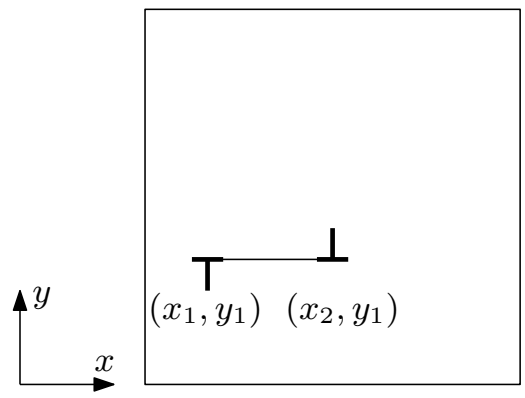

(b)

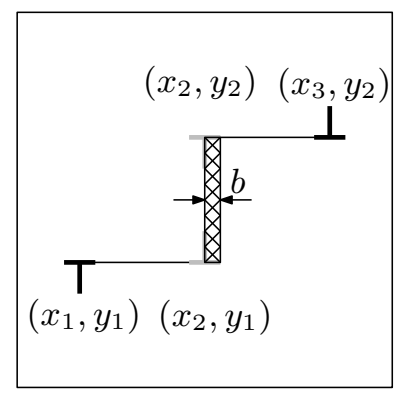

(c)

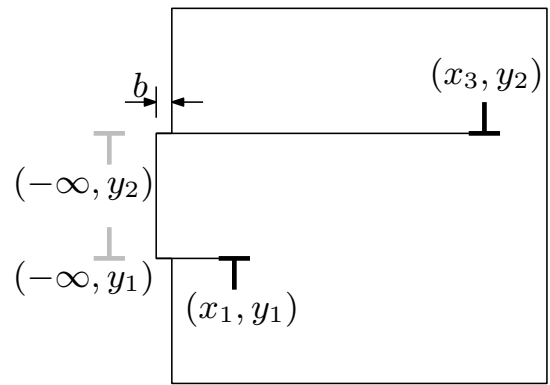

Figure 3: (a) Sketch of a rectangular domain containing an edge dislocation dipole on a horizontal slip plane, showing the slip discontinuity between the dislocations (solid line). (b) Displacement discontinuities after the positive dislocation at $\left(x_{2}, y_{1}\right)$ climbs to $\left(x_{2}, y_{2}\right)$ and then glides to $\left(x_{3}, y_{2}\right)$. The solid lines are slip discontinuities while the hashed region is interpenetration of matter over a distance $b$. (c) Our approximate method using pair dislocations at infinity.

at either end of the climb path. Further, such displacement corrections need to be added for every climb event in the simulation history in order to ensure correct termination of the slip traces. This approach has been adopted in several recent publications (Davoudi et al., 2012; Ayas et al., 2012) that consider combined glide and climb in 2D dislocation dynamics. This method is illustrated in Fig. 3b where the expression for the correction field for the climb step shown is

$$
\tilde{u}_{x}^{\text {corr }}=-\frac{b}{4} \operatorname{sign}\left(\left(x-x_{2}\right)\left(y-y_{1}\right)\right)+\frac{b}{4} \operatorname{sign}\left(\left(x-x_{2}\right)\left(y-y_{2}\right)\right), \quad \tilde{u}_{y}^{\text {corr }}=0
$$

While this approach is undoubtedly more accurate under general loading conditions, we have adopted a rather simpler approach that eliminates the need to keep track of the climb history by exploiting special features of the geometry we are simulating, described in Section 3.1. We assume that every dislocation inside the simulation domain is paired with an opposite signed dislocation at $-\infty$, as measured along the slip plane coordinate, and that a dislocation and its dipole pair climb together. In such case, the correction field to be introduced when a dislocation climbs corresponds to the sum of the eigenstrains of a pair of opposite signed dislocations at either end of the climb path at infinity (see Fig. 3c). The corresponding correction term for the $(\sim)$ displacement field is

$$
\tilde{u}_{x}^{\text {corr }}=-\frac{b}{4} \operatorname{sign}\left(y-y_{1}\right)+\frac{b}{4} \operatorname{sign}\left(y-y_{2}\right), \quad \tilde{u}_{y}^{\text {corr }}=0
$$

This approach eliminates the book keeping required to keep track of every climb event in the history of the simulation, but introduces additional slip discontinuities that could lead to spurious effects. However, it turns out that neither of the two approaches above have any effect on the computed stress and strain fields, provided the simulation domain is convex and none of the slip planes are allowed to intersect the boundary segments where displacements are prescribed. It must be noted that this is no longer true for other types 
of boundary conditions such as periodic or no-slip boundary conditions used in some of the above publications and it is necessary to account for the complete history dependent ( ) fields of the climbing dislocations.

For a domain subjected to mixed traction/displacement boundary conditions, as in Fig. 2a, the governing equations for the $\left(^{\wedge}\right)$ fields may be written as

$$
\nabla \cdot \hat{\boldsymbol{\sigma}}=\mathbf{0}, \quad \hat{\boldsymbol{\sigma}}=\mathbf{C}: \hat{\boldsymbol{\epsilon}}, \quad \hat{\boldsymbol{\epsilon}}=\frac{1}{2}\left(\nabla \hat{\mathbf{u}}+\nabla \hat{\mathbf{u}}^{T}\right)
$$

where $\mathbf{C}$ denotes the tensor of elasticity, and subjected to the boundary conditions

$$
\hat{\boldsymbol{\sigma}} \cdot \mathbf{n}=\mathbf{t}_{0}-\tilde{\boldsymbol{\sigma}} \cdot \mathbf{n} \quad \text { on } \partial V_{t}, \quad \hat{\mathbf{u}}=\mathbf{u}_{0}-\tilde{\mathbf{u}} \quad \text { on } \partial V_{u}
$$

where $\mathbf{t}_{0}$ and $\mathbf{u}_{0}$ are the prescribed tractions and displacements respectively on complementary regions $\partial V_{t}$ and $\partial V_{u}$ of the domain boundary $\partial V$. The above equations are solved using the finite element method. In particular, the same finite element mesh consisting of bilinear quadrilateral elements is used for the solution of both the diffusion and elasticity boundary value problems.

The glide velocity of a dislocation, $v_{g}^{i}$, is assumed to be proportional to the glide component of the Peach-Koehler force, i.e.

$$
v_{g}^{i}=f_{g}^{i} / B_{g}
$$

where the phonon drag coefficient $B_{g}$ is usually a linearly increasing function of the temperature. A theoretical estimate of the drag factor from Hirth and Lothe (1968) gives

$$
B_{g}=\frac{3 k T}{b^{2} c_{s}}
$$

where $c_{s}$ is the speed of shear waves in the material. In the simulations, we use a linear scaling for the drag factor of the form $B_{g}(T)=B_{g}^{0} T / T_{0}$ where $B_{g}^{0}$ is an experimentally determined value of the drag factor at a reference temperature, $T_{0}$. Frank-Read sources, idealized as point sources, are distributed randomly on every slip system. They can nucleate dislocation dipoles when the glide Peach-Koehler force on them exceeds a critical value $\tau_{\text {nuc }} b^{i}$ over a critical nucleation time. Further, gliding dislocations can be pinned by randomly distributed point obstacles and subsequently unpinned when the glide Peach-Koehler force exceeds the pinning strength of the obstacles. However, at finite temperatures, there is a possibility for dislocations to bypass obstacles at stresses below the athermal strength of the obstacles by some thermally activated mechanism such as cross-slip. In our high temperature formulation we allow for the possibility of thermally activated bypass of obstacles at sub-critical values of the Peach-Koehler force using a probabilistic formulation proposed by Frost and Ashby (1982) in the context of determining the drift velocity of a dislocation in a regular array of obstacles. Based on their analysis, at the end of each glide increment we allow for a dislocation to bypass an obstacle with a probability given by

$$
p_{\text {act }}=\exp \left[-\frac{\Delta F}{k T}\left(1-\frac{\left|f_{g}^{i}\right|}{\tau_{\text {obs }} b^{i}}\right)\right]
$$


where $f_{g}^{i}$ is the glide component of the Peach-Koehler force on the pinned dislocation and $\Delta F$ is the activation energy required to overcome the obstacle. Values for the latter are expected to range from $0.2 \mu b^{3}-2 \mu b^{3}$ for weak to strong obstacles. The reader is referred to the paper by Van der Giessen and Needleman (1995) for additional details about the constitutive rules used in two-dimensional glide simulations.

\section{Application to Creep}

Creep in single crystals is an ideal application of the coupled discrete dislocation and vacancy diffusion framework. Indeed, both time-scale and length-scale separations hold. First, the applied overall stress is typically lower than the yield stress so that quasiequilibrium dislocation configurations are attained granted sufficient time. Second, significant variations in vacancy concentration occur between the bulk, where the temperaturedependent equilibrium concentration $C_{0}(T)$ prevails with local perturbations due to vacancy consumption/production from climbing dislocations, and the boundary where a traction-dependent chemical potential prevails. Hence the gradients in $C$ occur over a length scale that is comparable with the specimen dimensions. In addition, the developed framework is ideal to investigate creep deformation in small structures where classical (visco)plasticity formulations may break down.

To set the stage, Fig. 4 depicts a typical creep curve under uniaxial loading. For an

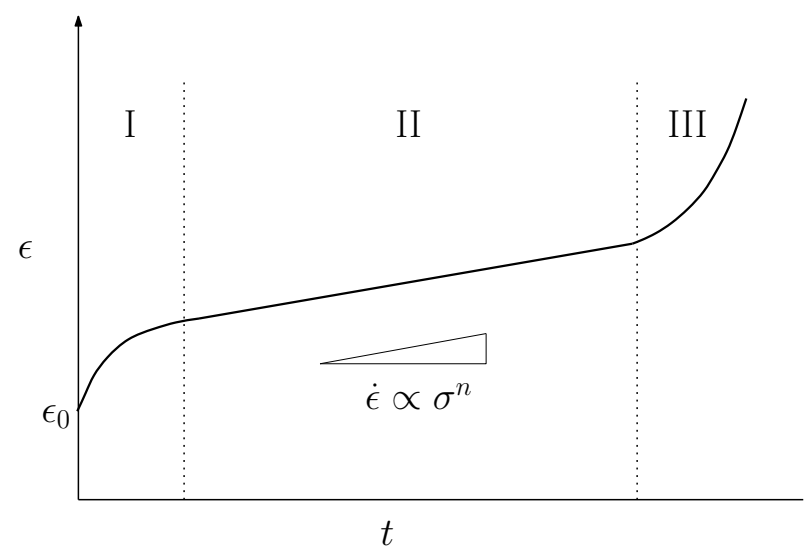

Figure 4: Sketch of a typical creep curve in crystalline materials.

imposed creep stress $\sigma$, the evolution of the strain $\epsilon$ with time $t$ shows three distinct stages. The initial offset strain $\epsilon_{0}$ is the instantaneous elastic strain due to the imposed stress. Stage I, also known as primary creep, denotes the transient during which the creep strain rate $\dot{\epsilon}$ decreases with time due to strain hardening. The design-limiting part of the creep curve is the steady state stage II in which the strain rate remains approximately constant as the result of a dynamic balance between strain hardening and thermal recovery processes. The final stage of accelerated deformation (tertiary creep) is due to damage processes such as cavitation and/or strain localization. The creep rates reported in the literature (e.g. Fig. 1) are the steady state secondary creep rates which typically follow 
a power law of the form $\dot{\epsilon} \propto \sigma^{n}$. Values of the power law exponent from experiments on bulk polycrystals fall in the range 3-8 with values larger than 5 being more typical (Frost and Ashby, 1982).

\subsection{Problem Definition and Simulation Approach}

We examine the creep behavior of plane strain single crystalline specimens of the type sketched in Fig. 5. The sample is loaded in tension along the $x_{1}$ direction by applying

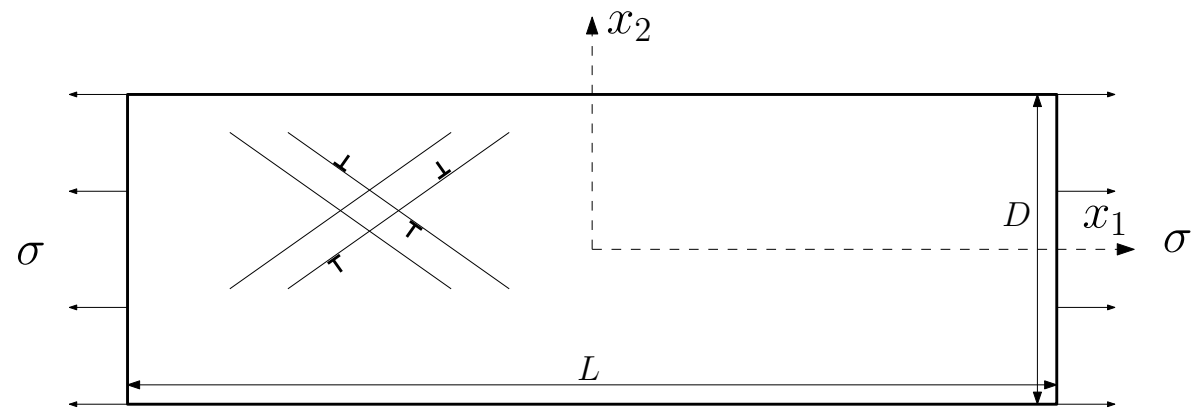

Figure 5: Schematic sketch of the plane strain tension specimen used in the creep computations.

uniformly distributed tractions $\sigma$ on the two end faces while the lateral boundaries of the specimen are traction free. A symmetric double slip arrangement is assumed with the two slip systems oriented at $\pm 35.25^{\circ}$ with respect to the tensile direction. The arrangement considered may correspond to an FCC single crystal oriented such that the plane of analysis coincides with the (110) crystallographic plane and loaded along the $\langle 001\rangle$ direction. The two slip systems considered correspond to the $\langle\overline{1} 12\rangle$ and $\langle 1 \overline{1} 2\rangle$ slip directions with an included angle of approximately $109.5^{\circ}$. Rectangular specimens are considered with an aspect ratio $L / D=3$ and sizes of the order of several microns. Initially, the crystal contains a distribution of dislocations with an average density of $120 \mu \mathrm{m}^{-2}$, point sources with a density of $150 \mu \mathrm{m}^{-2}$ and point obstacles with a density of $600 \mu \mathrm{m}^{-2}$. Some simulations are also performed with an order of magnitude smaller values for the initial densities in order to assess the sensitivity of the simulation results to initial conditions. The dislocations, sources and obstacles are distributed at random on the two slip systems considered. As in most previous discrete dislocation plasticity analyses, not all atomiclevel slip planes are represented within a slip system. Instead, potential slip planes are included on each system with a uniform spacing of $20 \mathrm{~b}$ between them. The random initial dislocation structure is assigned in such a way that the net Burgers vector in the specimen vanishes. The point sources represent Frank-Read sources whose nucleation strengths follow a normal distribution with average strength $\tau_{\text {nuc }}$ and standard deviation $\tau_{\text {sd }}$. The vacancy concentration field $C(\mathbf{x})$ is initialized such that it corresponds to the equilibrium (steady state) vacancy field in the sample of Fig. 5 subjected to the tensile tractions $\sigma$ at $x_{1}= \pm L / 2$ and traction free conditions at $x_{2}= \pm D / 2$.

The dislocations in the sample can glide on their respective slip planes in accordance with the Peach-Koehler force and the associated glide mobility law described in section 2.5. 
The dislocations can also climb to a neighboring slip plane according to the climb mobility law established in section 2.2, equation (24). Thus, unlike the glide process, dislocations climb between the slip planes in discrete steps set by the slip plane spacing. The climb distances of each dislocation, determined according to (24), are tracked at each time step. When the climb distance of a dislocation exceeds the slip plane spacing, the dislocation is moved from its original slip plane to the new slip plane. The production/consumption of vacancies as a result of the climb motion are used to calculate the vacancy source/sink term $C_{\text {src }}$ that enters equation (32) for the evolution of the vacancy field. A positive climb step equal to the slip plane spacing of $d_{\text {slp }}$ requires the absorption of $N_{v}$ vacancies into the dislocation core given by

$$
N_{v}=\frac{d_{\mathrm{slp}} b^{2}}{\Omega}
$$

Negative climb, on the other hand, requires the nucleation of the same number of vacancies with the associated atoms being absorbed into the dislocation core. Thus, negative climb of a dislocation occurs only when the local stresses are high enough to nucleate vacancies according to the energy criterion (Raabe, 1998)

$$
\sigma^{i} b^{3}=E_{f}
$$

where $\sigma^{i}$ is the tensile stress along the Burgers vector direction (excluding the self stress) at the location of dislocation $i$.

As remarked previously, time scale separation holds in this creep problem. Hence, we use an adaptive time stepping scheme. Dislocation nucleation and glide processes are modeled using a fixed time step $t_{\mathrm{gl}}=0.5 \mathrm{~ns}$. On the other hand, the vacancy diffusion and dislocation climb processes are simulated using a much larger time step $t_{\mathrm{cl}}(T)$ whose value strongly depends on temperature. A conservative estimate of the climb time step is made as $t_{\mathrm{cl}}=10^{-3} t_{\mathrm{est}}$, where $t_{\mathrm{est}}$ is an estimated time taken for a dislocation to climb a distance equal to the slip plane spacing. For the latter, an estimate of the climb velocity is made using equation (24) assuming $C=C_{0}$ and $f_{c}=100 \sigma b$ to allow for local stress concentrations within the sample. Creep simulations performed using different values of $t_{\mathrm{cl}}$ indicate the results are insensitive to the choice of the time step as long as $t_{\mathrm{cl}} \leq 10^{-2} t_{\text {est }}$. The iterative strategy used to perform the creep simulations is detailed below.

(i) The time step, $\mathrm{d} t$, is initialized as the glide time step $t_{\mathrm{gl}}$ at the beginning of the simulations.

(ii) The microstructure is initialized with randomly distributed dislocations, sources and obstacles according to their specified densities. Subsequently, glide simulations at zero stress are performed to relax the initial microstructure so that the dislocations attain local equilibrium positions.

(iii) Uniform displacements $\pm U / 2$ are applied on the two faces at $x_{1}= \pm L / 2$ while the lateral faces $x_{2}= \pm D / 2$ are taken to be traction free. The linear elastic boundary value problem, described in section 2.5 , is solved to obtain the stress and displacement fields in the sample. The average tensile stress and strain for the specimen are 
calculated as

$$
\sigma=\frac{1}{D} \int_{-D / 2}^{D / 2} \sigma_{11}\left( \pm L / 2, x_{2}\right) \mathrm{d} x_{2}, \quad \epsilon=\frac{U}{L}
$$

An iterative procedure is used to update the value of $U$ until the computed value of the average tensile stress equals the desired creep stress $\sigma$.

(iv) The Peach-Koehler forces on the dislocations are computed using equation (11). The dislocation positions are updated using the glide components of the PeachKoehler force and the glide mobility law (42). The constitutive rules for short range interactions between dislocations and obstacles described in section 2.5 are used during the glide steps to determine the new positions of the dislocations.

(v) Steps (iii)-(iv) are repeated until the average axial strain $\epsilon$ attains a steady state value. This may correspond to the dislocations in the sample reaching local equilibrium positions, such as stuck at obstacles, so that the glide activity in the system ceases and the overall strain rate nominally vanishes. In practice, dislocation activity never completely stops in a large dynamical system and an alternative criterion is needed to detect steady state conditions. In our simulations steady state conditions are considered to be attained when the average strain rate remains zero (within a specified tolerance) over a period of 100 glide increments.

(vi) When the steady state is reached, the dislocations are frozen at their current positions and the time step $\mathrm{d} t$ is switched to the climb time step $t_{\mathrm{cl}}$. The diffusion boundary value problem, described in section 2.2, is solved using the finite element method. At the beginning of the simulation, the vacancy field is initialized using the equilibrium distribution consistent with the imposed boundary tractions and the temperature. The initial conditions for any subsequent step correspond to the vacancy field at the end of the previous time step. The contribution to the total strain as a result of the diffusive flux of vacancies through the boundaries is calculated as

$$
\epsilon_{d}=-\int_{0}^{t} \frac{\mathrm{d} t}{L D} \int_{-D / 2}^{D / 2} \mathbf{J}\left(x_{1}= \pm L / 2, x_{2}\right) \cdot \mathbf{n} \mathrm{d} x_{2}
$$

(vii) The climb distances of the dislocations at the end of each increment are evaluated using equation (24). When the climb distance of any of the dislocations in the sample reaches the slip plane spacing, the dislocation is moved from its original slip plane to the new slip plane. Positive and negative climb are treated differently according to the energy based criterion for vacancy nucleation, equation (46). The production term in the governing equation for $C$ is estimated on a per element basis by identifying the elementary volume in equation (45) with the elements in the finite element grid.

(viii) The time step $\mathrm{d} t$ is switched back to the glide time step after the first climb event and the glide steps (iii)-(iv) are repeated until a new steady state value of the strain $\epsilon$ is reached. 
Material properties for Aluminum are used in the simulations with Young's modulus $E=70 \mathrm{GPa}$, Poisson's ratio $\nu=0.33$ and Burger's vector modulus $b=0.25 \mathrm{~nm}$. The temperature dependence of the elastic moduli is ignored but the drag factor is taken to scale linearly with $T$ as $B=10^{-4} T / 300 \mathrm{~Pa} \mathrm{~s}$, where $B=10^{-4} \mathrm{~Pa}$ s is the value at $T=300 \mathrm{~K}$. A constant value of $\tau_{\text {obs }}=150 \mathrm{MPa}$ is used for the athermal strength of the point obstacles. However, thermally activated bypass of obstacles at sub-critical values of the Peach-Koehler force is modeled using the probabilistic criterion (44). A normal distribution of initial source strengths is assumed with average value and standard deviation $\tau_{\text {nuc }}=50 \mathrm{MPa}$ and $\tau_{\text {sd }}=10 \mathrm{MPa}$, respectively. As pointed out by Shishvan and Van der Giessen (2010) a distribution of nucleation times following the model of Benzerga (2008) is preferred, but here a constant value of $t_{\text {nuc }}=10 \mathrm{~ns}$ is assigned to all sources for simplicity. The material properties that enter the constitutive rules for vacancy diffusion are tabulated based on data from the literature, Table. 1 . Note that

\begin{tabular}{|c|c|c|}
\hline Property & Symbol & Value \\
\hline Melting Temperature & $T_{m}$ & $933 \mathrm{~K}$ \\
\hline${ }^{a}$ Atomic Volume & $\Omega$ & $16.3 \AA^{3}$ \\
\hline b,c Vacancy Relaxation Volume & $\Omega_{v}$ & $\sim 0 \AA^{3}$ \\
\hline${ }^{\mathrm{d}}$ Vacancy diffusion coefficient pre-exponential & $D_{0}$ & $1.51 \times 10^{-5} \mathrm{~m}^{2} / \mathrm{s}$ \\
\hline${ }^{\mathrm{d}}$ Vacancy formation energy & $E_{f}$ & $0.67 \mathrm{eV}$ \\
\hline dVacancy migration energy & $E_{m}$ & $0.61 \mathrm{eV}$ \\
\hline
\end{tabular}

Table 1: Material properties for Al used in the creep simulations. From references ${ }^{a}$ Hirth and Lothe (1968), ${ }^{\mathrm{b}}$ Schilling (1978), ${ }^{\mathrm{c}}$ Harrison and Wilkes (1972), ${ }^{\mathrm{d}}$ Freund and Heinloth (2002).

the formation volume for a vacancy in $\mathrm{Al}$ is very nearly equal to the atomic volume as determined from experiments (Schilling, 1978) and atomistic calculations (Harrison and Wilkes, 1972) so that the relaxation volume $\Omega_{v}$ is nearly zero. Since $\Omega_{v}$ is taken to vanish in the calculations the hydrostatic pressure has no effect on the vacancy chemical potential (12). Simulations investigating the effect of a non-zero relaxation volume will be reported elsewhere. Also, the length scale entering the production term in (30) is taken to be $l_{g}=100 \mathrm{~nm}$ A structured mesh is used with constant element size $\sim 100 \mathrm{~nm}$. The fact $l_{g}$ is comparable with the finite element size is for mere convenience and the results are not mesh-dependent.

\subsection{Simulation Results}

\subsubsection{Nominal yield stress}

We first examine the stress-strain response of a typical tensile specimen to determine the yield strength and post-yield behavior of the material. This was obtained by carrying out calculations at a constant imposed displacement rate of $\dot{U} / L=10^{4} \mathrm{~s}^{-1}$. The ratesensitivity of the material is weak enough to consider the flow stress levels representative of lower strain rate calculations as well. Fig. 6 shows a representative stress-strain response 
of a specimen of width $D=4 \mu \mathrm{m}$. The material exhibits an elastic-plastic behavior with a

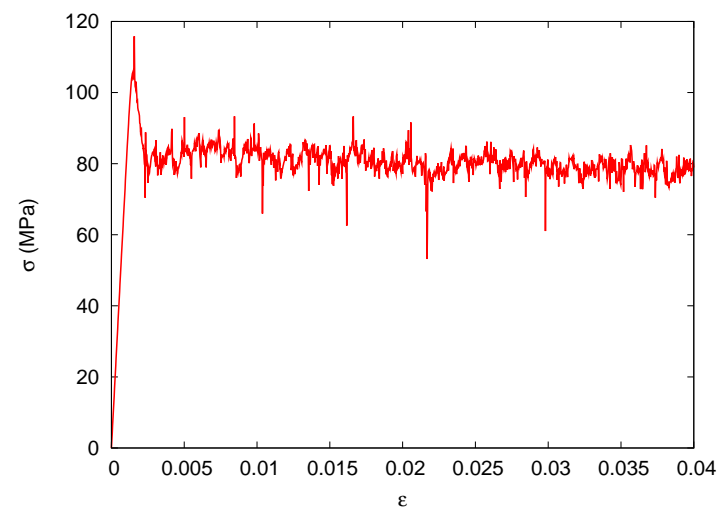

(a)

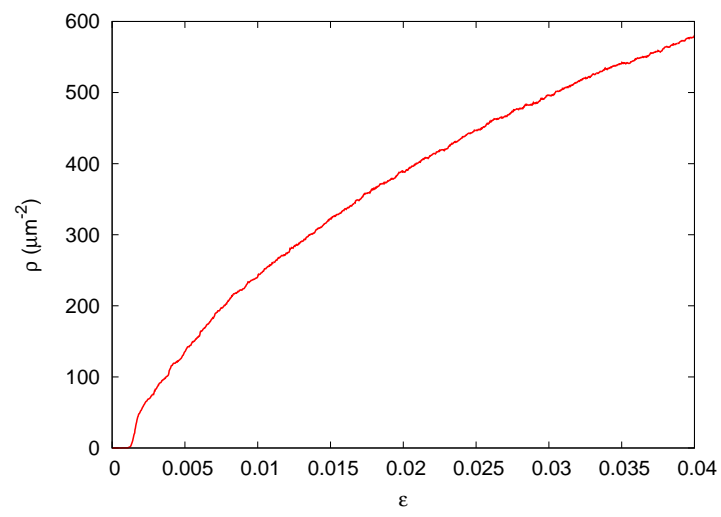

(b)

Figure 6: Evolution of the (a) flow stress $\sigma$ and (b) dislocation density $\rho$ for a computational specimen with $D=4 \mu \mathrm{m}$ subjected to a constant deformation rate $\dot{\epsilon}=10^{4} \mathrm{~s}^{-1}$.

lower yield stress of approximately $80 \mathrm{MPa}$, and no strain hardening consistent with prior simulations in the literature using the 2D discrete dislocation model. The upper yield stress of a given specimen is set by the chosen values of the average nucleation strength of the static Frank-Read sources. It is noted that there is minimal scatter in the value of the flow stress for different realizations of the initial source and obstacle populations due to the rather high values of the source and obstacle densities chosen. Examination of similar results for different values of $D$ within the range $2-8 \mu \mathrm{m}$ shows that there is no size dependence of the yield stress. The choice of high initial densities enables us to reduce the scatter in the simulation results and to isolate collective emergent behavior in a large dynamical system.

\subsubsection{Dislocation Creep}

Creep simulations were performed for single crystalline specimens of the type shown in Fig. 5 at several values of the absolute temperature, in the range $T=400-800 \mathrm{~K}$, and different values of the creep stress $\sigma$ below the yield stress of the specimen in tension. Assuming material properties of Aluminum, the chosen range of the temperature corresponds to $0.43-0.86 T_{m}\left(T_{m}=933 \mathrm{~K}\right)$.

Fig. 7 shows the creep response of the same computational specimen for different values of the creep stress $\sigma$ in the range $10-60 \mathrm{MPa}$ at temperature $T=400 \mathrm{~K} \approx$ $0.43 T_{\mathrm{m}}$. Fig. $7(\mathrm{a})$ shows the evolution of the axial strain $\epsilon$ as a function of time and Fig. 7(b) shows the evolution of the dislocation density with time. Note that, due to the rapid oscillation of the dislocation density about a well defined mean, the curves of $\rho$ vs. time have been smoothed out using the plotting software so that the different curves are clearly distinguishable in the figure. It can be seen from Fig. 7 that after an initial transient region, the dislocation density does not vary significantly with strain except at high values of the creep stress, unlike in the tensile simulation of Fig. 6. Steady 


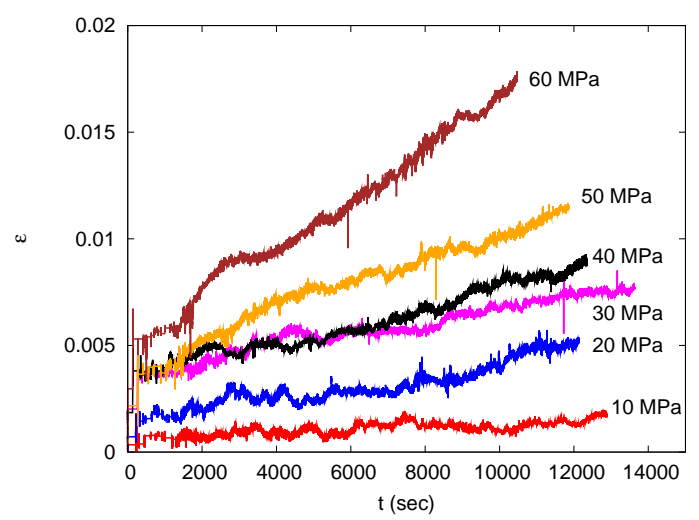

(a)

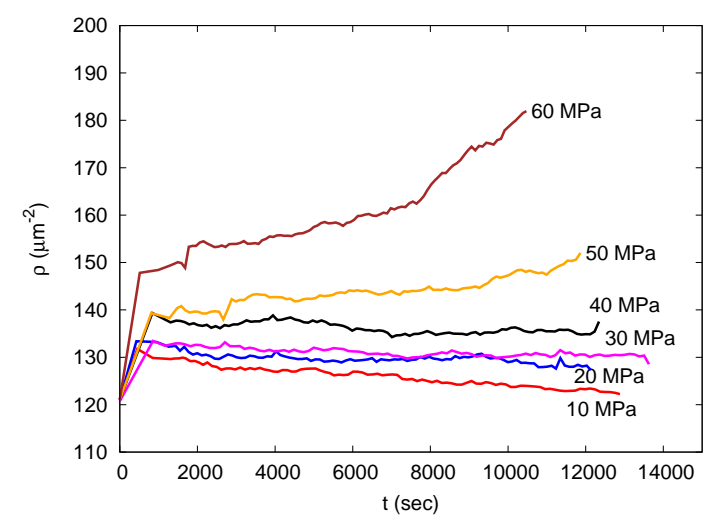

(b)

Figure 7: Creep response of specimens with $D=4 \mu \mathrm{m}$ at $T=400 \mathrm{~K}$ for different values of the creep stress $\sigma$ below the macroscopic yield stress: (a) strain $\epsilon$ vs. time $t$ and (b) dislocation density $\rho$ vs. $t$. The curves are labeled by the value of the creep stress $\sigma$.

state stage II creep (see Fig. 4) is characterized by more or less constant values of the microstructural variables (Frost and Ashby, 1982), which in the case of our single crystal simulations correspond to the dislocation density. Hence, we may consider the part of the creep curves after the transient in Fig. 7 to correspond to the steady state creep regime in Fig. 4. However, closer examination of the initial transient region in Fig. 7(a) shows a serrated curve with alternating regions of rapid straining followed by large plateau regions with no accumulation of strain unlike a smooth transient of the type shown in Fig. 4. This is probably an artifact of the specific initial conditions chosen for the system. For instance, sufficient time must elapse of the vacancy flow into the dislocation cores to cause dislocation climb, which means that the mean time between climb events is large initially, before a steady state is reached for the rate at which glide and climb events occur in the system. Finally, we also note that the final stage of accelerated deformation (stage III creep in Fig. 4) is a result of damage processes in the system such as cavitation, which we do not attempt to model using the present small strain framework. Also, limitations of computing time, especially at larger temperatures where it is necessary to use a much smaller climb time step $t_{\mathrm{cl}}$, limits our simulation efforts to steady state creep.

Fig. 8 shows similar creep curves for the $D=4 \mu \mathrm{m}$ specimen at higher values of the temperature $T=600 \mathrm{~K}$ and $T=800 \mathrm{~K}$, i.e., $0.63 T_{\mathrm{m}}$ and $0.86 T_{\mathrm{m}}$ respectively. Notice that the simulation results are shown for much shorter durations of time compared to the simulations at $T=400 \mathrm{~K}$. This is due to the fact that the climb time step $t_{\mathrm{cl}}$ is a rapidly decreasing function of the temperature, although even at $T=800 \mathrm{~K}, t_{\mathrm{cl}}$ is at least three orders of magnitude larger than the glide time step $t_{\mathrm{gl}}=0.5 \mathrm{~ns}$. Despite the different ranges of the time axis, the creep curves at the different temperatures are qualitatively similar with an extended steady state regime following a short transient stage. The creep strain rates $\dot{\epsilon}$ are significantly higher at higher temperatures as expected. The evolution of the dislocation density associated with the results in Fig. 8 (not shown) are very similar to that in Fig. 7(b) with similar values for the steady state dislocation density. Also, the 


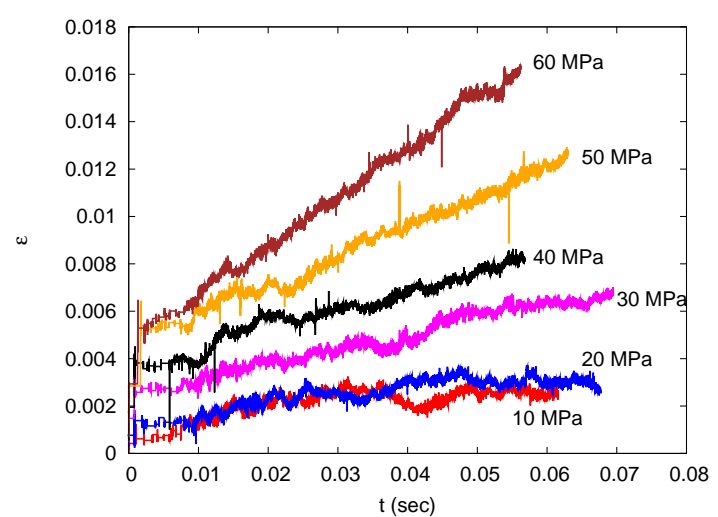

(a)

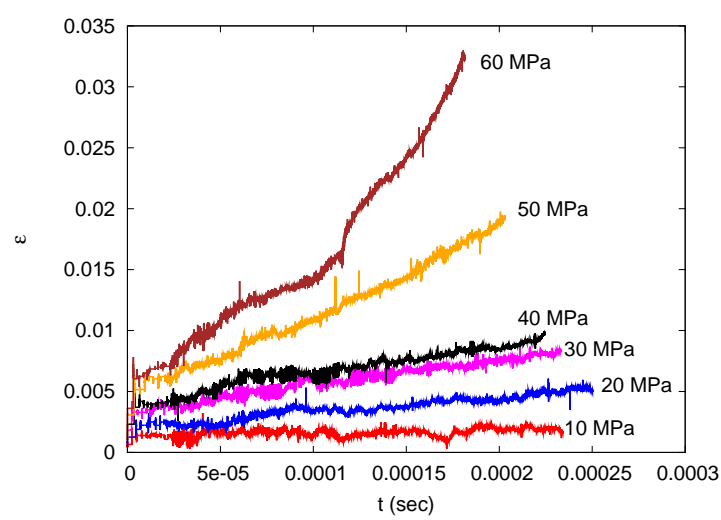

(b)

Figure 8: Creep curves for the $D=4 \mu \mathrm{m}$ specimen at (a) $T=600 \mathrm{~K}$ and (b) $T=800 \mathrm{~K}$ and different values of the creep stress. The curves are labeled by the value of the creep stress $\sigma$.

order of magnitude of the creep rates predicted by our simulations, estimated by a linear least squares fit to the steady state creep curves, agrees well with the experimental creep rates in $\mathrm{Al}$ summarized in Fig. 1. However, the two are not directly comparable due to the fact that deformation mechanism map of Fig. 1 is based on data on polycrystals while the simulations are done on single crystals.

A better approach to validate the model predictions is to consider plots for the predicted creep rate as a function of temperature. The functional dependence of the steady state creep rates on temperature is expected to follow an Arrhenius equation of the form

$$
\dot{\epsilon}=\dot{\epsilon}_{0} \exp \left(-\frac{Q}{k T}\right)
$$

where $\dot{\epsilon}_{0}$ is a reference creep rate and $Q$ denotes the activation energy for creep. Fig. 9 shows the variation of the steady state creep rate as a function of the reciprocal temperature for various values of the creep stress. The negative slope of the activation plot on this semi-log scale yields the activation energy for creep predicted by the simulations. It is remarkable that irrespective of the applied stress, a roughly constant slope is obtained for the activation plot. What is of particular importance is that the predicted value of the activation energy is around $120 \mathrm{~kJ} / \mathrm{mol}(1.24 \mathrm{eV})$, which is approximately equal to the activation energy for self-diffusion, $E_{s}=E_{f}+E_{m}$, where $E_{f}$ and $E_{m}$ are the vacancy formation and migration energies, respectively, as defined in previous sections (also see Table. 1). This result shows that diffusion assisted dislocation climb is indeed the microscale mechanism controlling the creep process and serves as an indirect validation of our simulation approach.

\subsubsection{Diffusional Creep}

Apart from the strain $\epsilon=U / L$ shown in Figs. 7-8, which mainly results from dislocation glide on the two slip systems, part of the total creep strain is due to the contribution from 


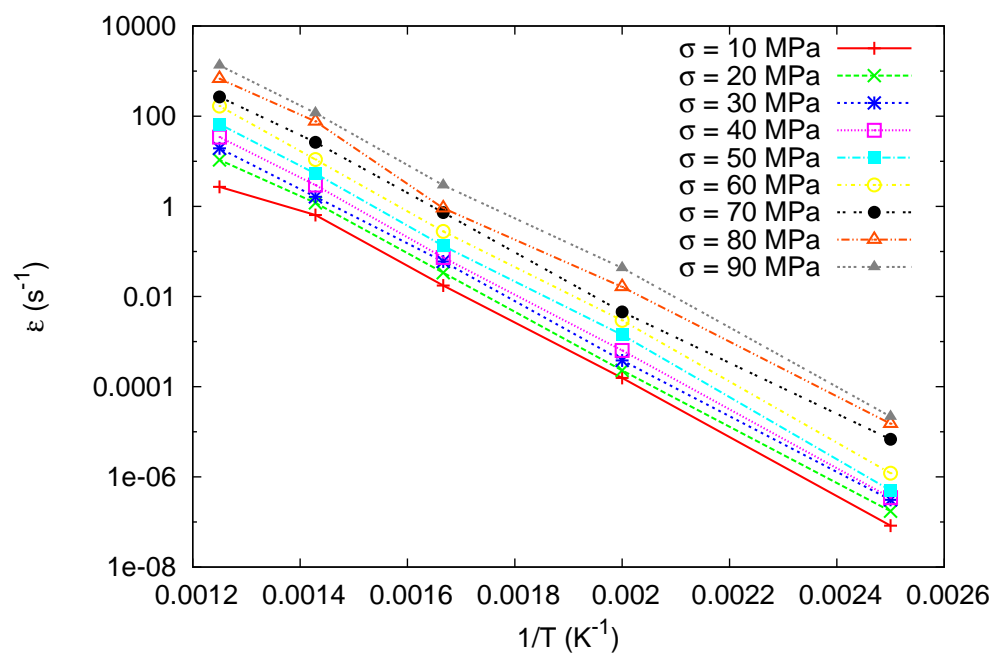

Figure 9: Plot of the creep strain rate as a function of the reciprocal temperature for various values of the creep stress.

mass transport due to the flux of vacancies. In the case of the tensile sample of Fig. 5, the boundaries at $x_{1}= \pm L / 2$ have a higher chemical potential for vacancies compared to the free boundaries at $x_{2}= \pm D / 2$, and this leads to a continuous flux of vacancies from the former to the latter. Since the diffusive flux of mass is opposite to the flux of vacancies, this leads to inelastic extension of the bar in the direction of tension. The corresponding strain $\epsilon_{d}$ is given by equation (48) and represents Nabarro-Herring creep. Fig. 10 shows an example of the typical contours of vacancy concentration $C(\mathbf{x})$ in the specimen. The contours in Fig. 10 correspond to the instantaneous profile of vacancies at $t=0.05 \mathrm{~s}$ in the $D=4 \mu \mathrm{m}$ specimen at $T=600 \mathrm{~K}$ and subjected to a creep stress $\sigma=40 \mathrm{MPa}$. One can clearly see from Fig. 10 that the gradients in $C$ and hence the flux of vacancies is directed away from the tensile boundaries on the right and left and towards the free boundaries at the top and bottom.

Fig. 11(a) and (b) show the evolution of the Nabarro-Herring creep strain $\epsilon_{d}$, computed using equation (48), as a function of time at $T=600$ and $800 \mathrm{~K}$ corresponding to the creep curves in Fig. 8. Notice that the magnitudes of $\epsilon_{d}$ are several orders of magnitude smaller than the strain $\epsilon$ due to dislocation creep at all times. Thus the simulations show that dislocation creep is the dominant mode of deformation for the considered ranges of temperature and creep stresses. However, when the creep stresses are small enough so that no dislocation activity is possible, the only remaining contribution to the total strain rate is due to the diffusional creep shown in Fig. 11.

\subsubsection{Stress Dependence of the Creep Rate}

The creep strain rate is usually related to the stress through a power law relationship of the form $\dot{\epsilon} \propto \sigma^{n}$, where $n$ depends on the dominant mode of creep. Here, we examine the scaling of the creep strain rate as a function of the stress obtained from the simulations. Fig. 12 shows the variation of the creep strain rate as a function of the stress at $T=$ 


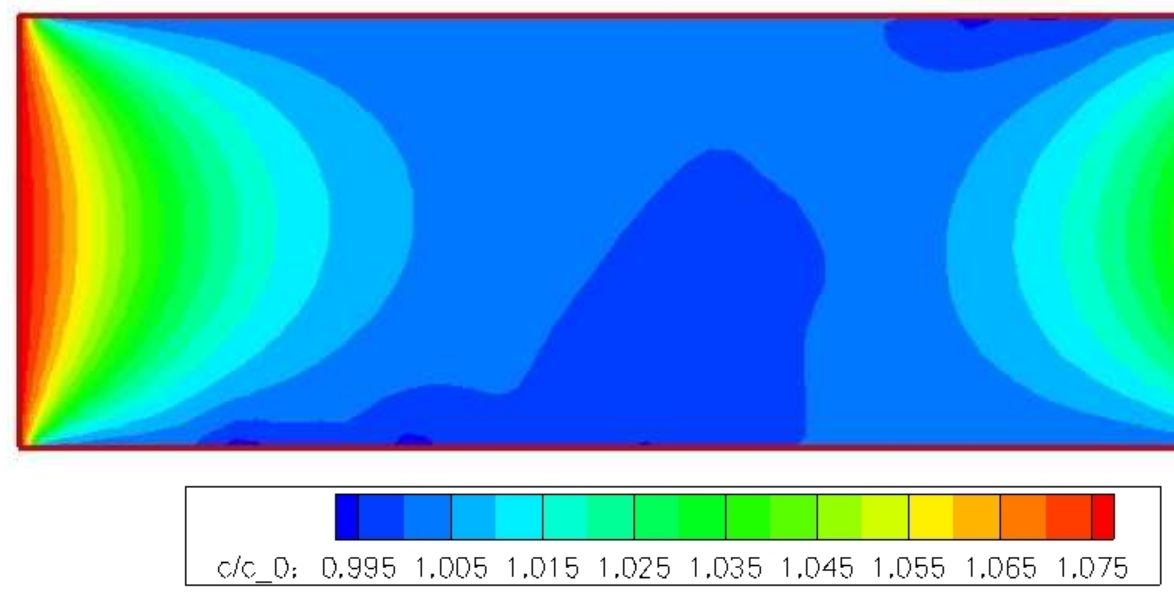

Figure 10: Contours of vacancy concentration $C$ normalized by the equilibrium vacancy concentration $C_{0}$ in the $D=4 \mu \mathrm{m}$ specimen at $T=600 \mathrm{~K}, t=0.05 \mathrm{~s}$ and creep stress $\sigma=40 \mathrm{MPa}$.

400K, plotted on a log-log scale. The resolved shear stress on the slip systems $\tau=f_{s} \sigma$, normalized by the value of the shear modulus $\mu$, are shown along the $x$-axis for ease of comparison with deformation mechanism maps such as Fig. 1. The Schmid factor, $f_{s}$, is equal to 0.47 for both the slip systems in the case of the symmetric slip system configuration considered. Average strain rates obtained from simulations on multiple realizations of the microstructure are shown along with the associated scatter. A minimum of three realizations have been considered for each case. Values of the strain rates $\dot{\epsilon}$ and $\dot{\epsilon}_{d}$ are calculated using a linear least square fit in the steady state region of the creep curves. Notice that the scatter is much lower for the diffusional creep rates $\dot{\epsilon}_{d}$ compared to the dislocation creep rates $\dot{\epsilon}$, reflecting the fact that the latter rates are set by the collective behavior of the discrete dislocations, which introduces some stochasticity. Fig. 12(a) also shows results for the dislocation creep rates obtained using an order of magnitude smaller values of the initial source and obstacle densities compared to the values of $\sim 10^{14} \mathrm{~m}^{-2}$ used in all other simulations. While the observed creep rates are smaller in the case of the lower initial densities, the scaling behavior for the strain rate with stress is qualitatively similar to the results at higher initial densities and exhibits the transition in the creep exponent from low to high stresses. This indicates that the simulation trends are not strongly dependent on initial conditions. Finally, we observe that the diffusional creep rates in Fig. 12(b) are much smaller than the creep due to dislocation glide and the scaling exponent for the stress is nearly equal to 1 .

Figs. 13 shows similar results for higher values of the temperature $T=600 \mathrm{~K}\left(0.64 T_{m}\right)$ and $T=800 \mathrm{~K}\left(0.86 T_{m}\right)$ respectively. Note that plots for $\dot{\epsilon}_{d}$ vs. time at these temperatures were qualitatively similar to Fig. 12(b), with a value of the stress exponent equal to unity, and are hence not shown here. The qualitative trends in Fig. 13 are similar as in the results at $T=400 \mathrm{~K}$ with $\dot{\epsilon}_{d} \ll \dot{\epsilon}$ and the exponent $n$ generally increasing to a large value for stresses larger than $\sim 0.001 \mu$. Also, the order of magnitude of the creep rates are in agreement with experimental values in Fig. 1 . A somewhat smaller value of $n \approx 5$ 


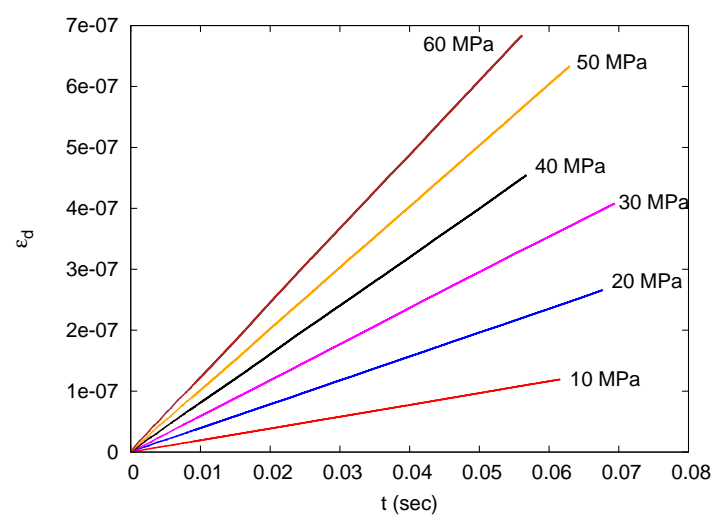

(a)

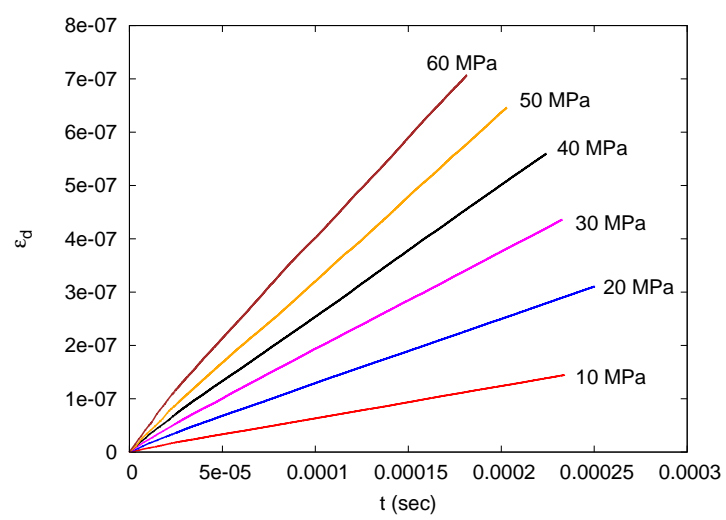

(b)

Figure 11: Diffusional creep strain $\epsilon_{d}$ as a function of time for the $D=4 \mu \mathrm{m}$ specimen at (a) $T=600 \mathrm{~K}$ and (b) $T=800 \mathrm{~K}$ and different values of the creep stress, corresponding to the creep curves of Fig. 8. The curves are labeled by the value of the creep stress $\sigma$.

is obtained at high stresses, compared to the value of $\sim 7$ obtained at $T=400 \mathrm{~K}$ in Fig. 12(a), while the low stress exponent $n$ ranges from $1-2$. In fact, a stress exponent in the range $5-7$ was also obtained in our simulations done at $T=500$ and $700 \mathrm{~K}$ (not shown), in excellent agreement with the experimentally reported range of the stress exponents. Also, a clear transition to a much smaller value of the stress exponent $\sim 1$ was observed towards lower creep stresses in all our simulations.

\subsubsection{Aspects of creep in small-scale specimens}

Unlike for bulk polycrystals, experimental data is not readily available for single crystal creep at small scales. However, recently Ng and Ngan (2007) have performed creep experiments on micron sized single crystalline Al columns manufactured using focussed ion beam milling subjected to nominally homogeneous compression using a flat tip nanoindenter. Unlike other experiments that examined the effect of specimen size on the flow strength at small scales (Uchic et al., 2004; Greer et al., 2005), their experiments were performed under load control rather than displacement control and the investigation was focused on the creep behavior of the pillars at room temperature. Their main finding was that creep curves for these pillars at high stresses exhibited a staircase like aspect with regimes of steady state creep interspersed with intermittent strain bursts. Such strain bursts have not previously been observed in creep experiments in bulk polycrystals. A similar behavior has also been observed in some of our DD simulations at high values of the creep stress below the yield stress of the specimens. Fig. 14 shows a qualitative comparison of the experimental creep data from Ng and Ngan (2007) with an example creep curve from our simulations that exhibited a similar staircase aspect. The experimental data in Fig. 14(a) correspond to cylindrical micropillars of diameter $6.3 \mu \mathrm{m}$ and

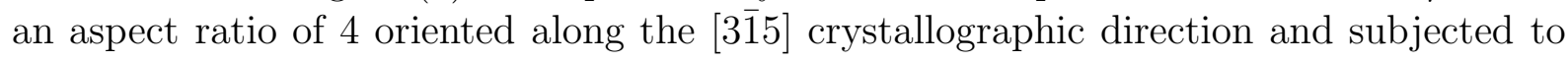
creep loading at various values of the stress for a duration of 300 seconds. The simulation data corresponds to a plane strain tension specimen with $D=4 \mu \mathrm{m}$ subjected to a creep 


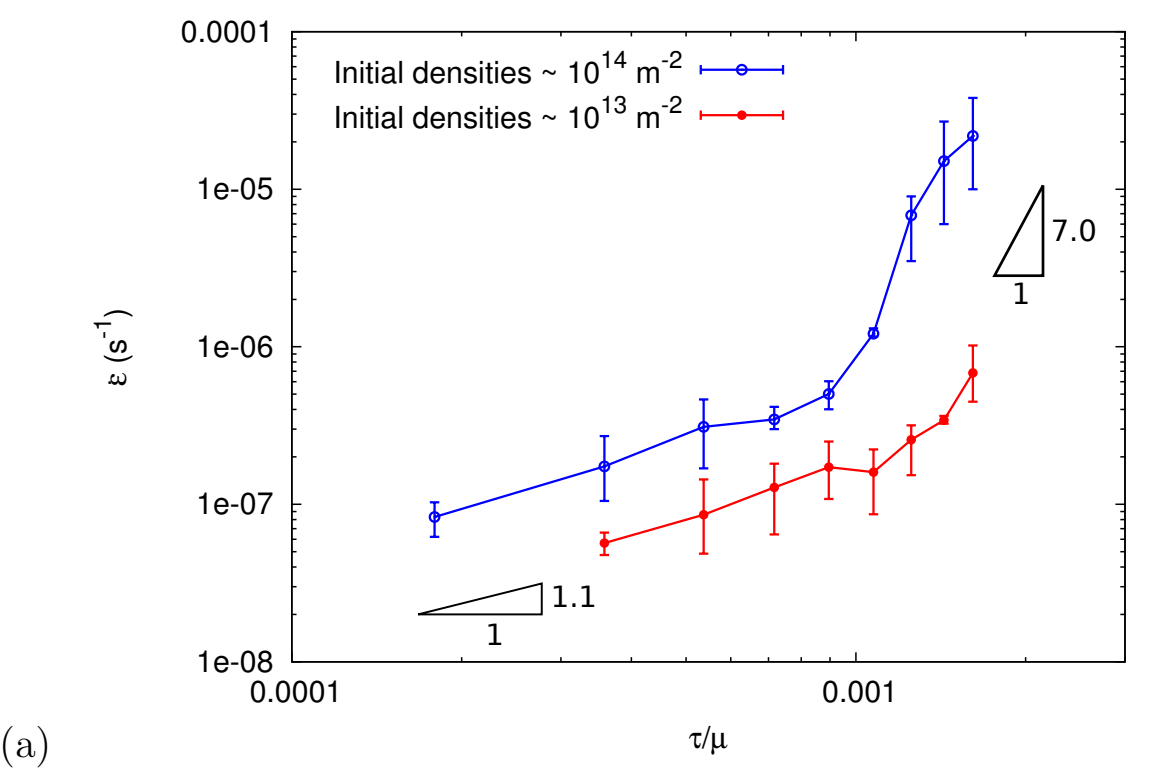

(a)

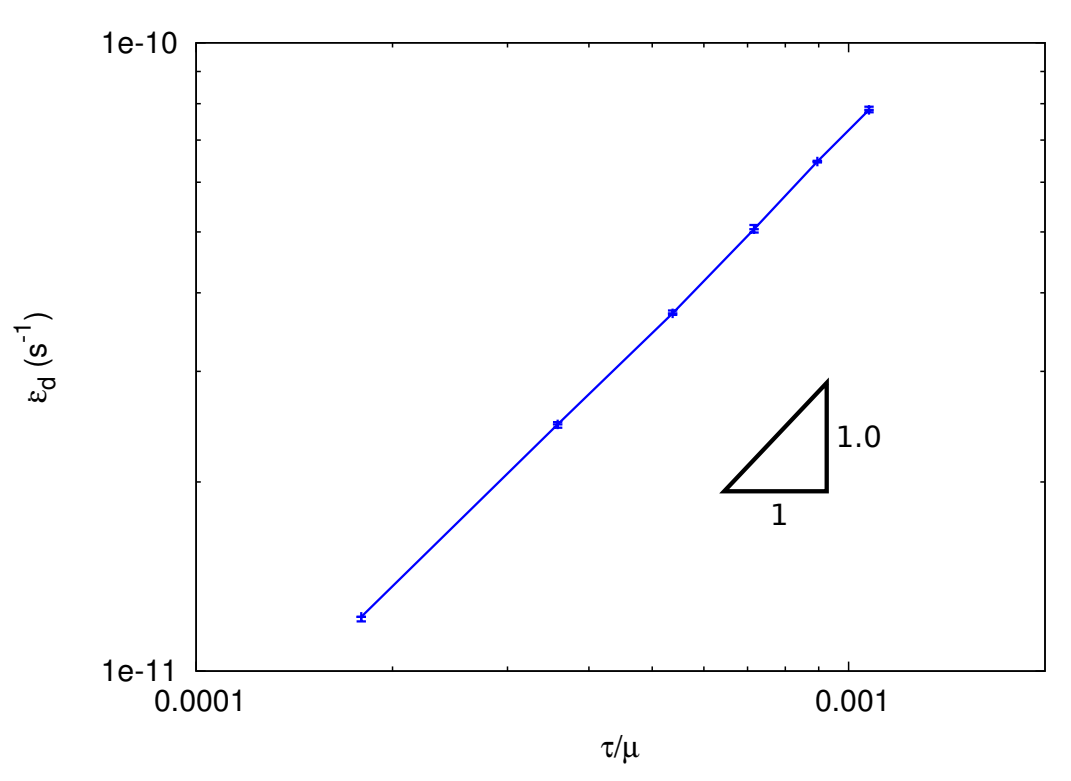

Figure 12: Variation of the creep strain rate as a function of stress obtained from the DD simulations at $T=400 \mathrm{~K}$ : (a) dislocation creep and (b) diffusion creep. The error bars denote the scatter in the predicted creep rates obtained from at least three realizations of the initial configurations of dislocations, sources and obstacles.

stress $\sigma=50 \mathrm{MPa}$. Based on microscopic examination of the deformed specimens, Ng and Ngan (2007) have concluded that the strain bursts are not the result of discrete nucleation events localized to a single slip plane and rather occur due to coordinated action of dislocation sources on multiple slip planes. Figs. 15(a) and (b) show the contours of total slip, $\Gamma$, in the computational specimen during two time intervals corresponding to $t=2500-3000 \mathrm{~s}$ and $t=3000-3500 \mathrm{~s}$ respectively. Note that the latter time interval encompasses the duration of the strain burst in Fig. 14(b). Total slip $\Gamma$ for the time 


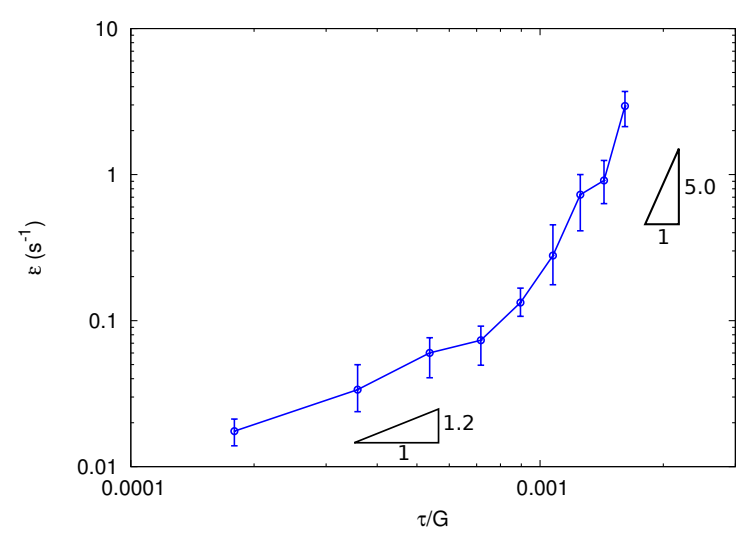

(a)

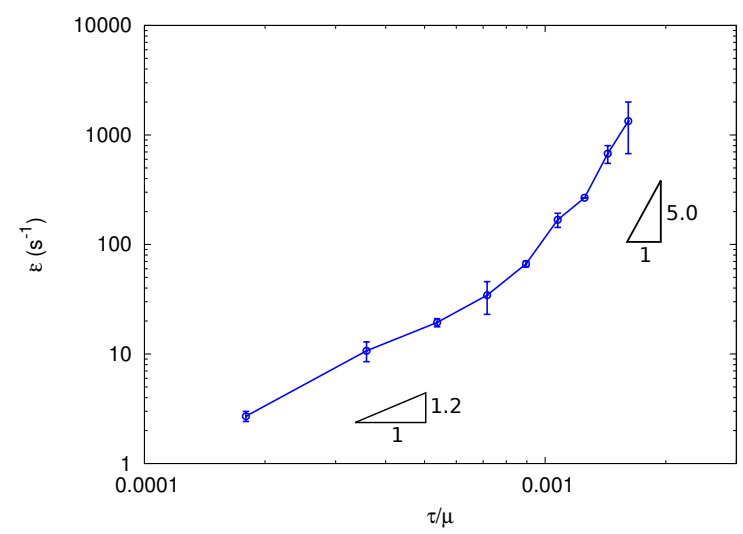

(b)

Figure 13: Variation of the creep strain rate as a function of stress obtained from the DD simulations at (a) $T=600 \mathrm{~K}$ and (b) $T=800 \mathrm{~K}$. The error bars denote the scatter in the predicted creep rates obtained from at least three realizations of the initial configurations of dislocations, sources and obstacles.

interval $t_{1}-t_{2}$ is defined as $\Gamma=\int_{t_{1}}^{t_{2}} \dot{\Gamma} \mathrm{d} t$, where $\dot{\Gamma}$ is a measure of the point-wise slip rate defined by $\dot{\Gamma}=\sum_{\kappa}\left|\dot{\gamma}^{\kappa}\right|$, with $\dot{\gamma}^{\kappa}=m_{i}^{\kappa} \dot{\epsilon}_{i j}^{s} n_{j}^{\kappa}$. Here, $\mathbf{m}$ and $\mathbf{n}$ denotes the slip direction and slip plane normal respectively for slip system $\kappa$ and $\dot{\boldsymbol{\epsilon}}^{s}$ is a smooth strain rate field introduced in each finite element and computed by differentiating the total displacement rate field $\dot{\mathbf{u}}$ in that element using the finite element shape functions. The slip contours in Fig. 15 show significantly higher slip activity during the strain burst compared to a similar time interval during the steady state creep regime. Further, it can be seen that slip activity during the strain burst is distributed over a band of slip planes in one of the two slip systems considered.

\section{Concluding Remarks}

A continuum physics based framework has been introduced for describing thermally activated, dislocation-mediated inelastic deformation processes. Both conservative glide and nonconservative climb motions of a large number of discrete dislocations are accounted for in the formulation. The latter involves (i) determining the long- and short-range dislocation interactions by representing the dislocations as line singularities in a finite elastic body; (ii) obtaining the stress-affected diffusion flux of point defects represented by a concentration field; and (iii) accounting for the interaction of point defects with dislocations, giving rise to a chemical osmotic force and a drag force on the dislocations, in addition to the climb component of the mechanical Peach-Koehler force. The computational framework consists of solving two staggered initial/boundary value problems: a linear elasticity problem and an unsteady diffusion problem. It enables a straightforward extension of discrete dislocation plasticity (Van der Giessen and Needleman, 1995) to high homologous temperatures. 


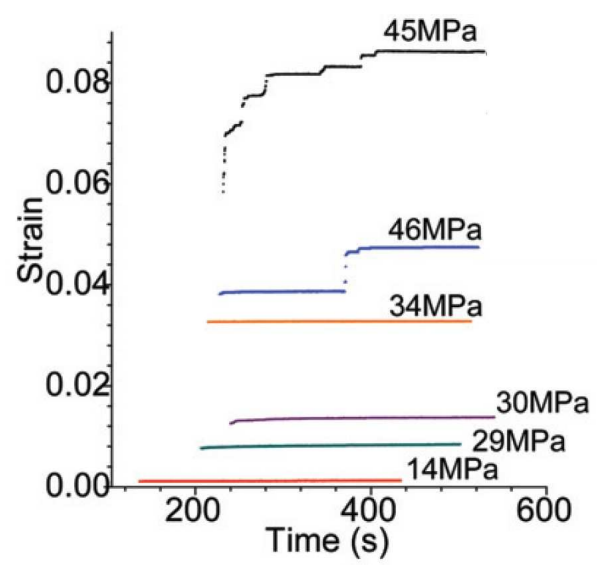

(a)

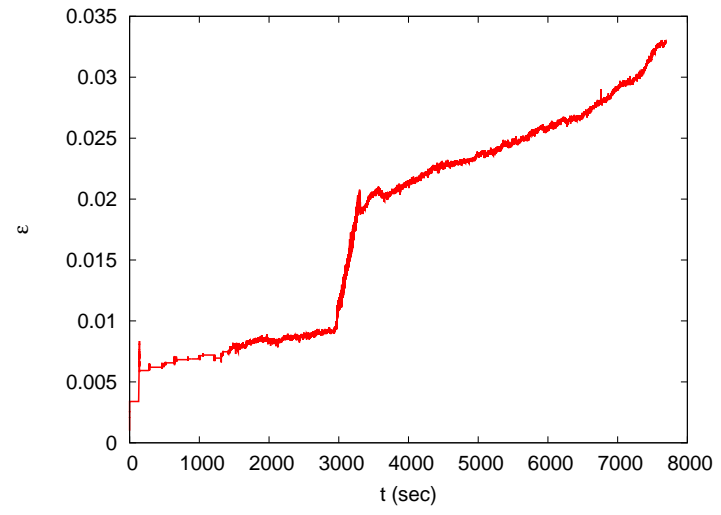

(b)

Figure 14: (a) Experimental data for creep of $6.3 \mu \mathrm{m}$ diameter Al single crystals, adapted from Ng and Ngan (2007). (b) Strain burst observed during a DD simulation of creep in a $D=4 \mu \mathrm{m}$ specimen subjected to tensile creep stress $\sigma=50 \mathrm{MPa}$.

Key to the feasibility of the staggered approach is time-scale separation. The challenge of the widely disparate time scales for dislocation glide and climb is overcome by postulating the existence of quasi-equilibrium configurations for the problems of interest. In practice, adaptive time stepping is implemented in the staggered approach with fixed glide time steps $(\sim n s)$ and temperature dependent climb time steps $(\sim m s)$. The glide steps are responsible for the rearrangement of dislocations between local equilibrium positions on their respective slip planes (stuck at obstacles, for instance) and consequently for most of the macroscopically observed strain. The climb process, occurring over a much longer time scale, sets the overall strain rate by determining the rate at which dislocations are able to bypass obstacles through thermal activation.

The equations that govern the unsteady diffusion problem were derived from a variational principle. Without the requirement of length scale separation, the governing equations are general and enable a direct determination of the climb velocity of each dislocation, as affected by the chemical and drag forces. Solutions to the general equations are useful, for example to investigate unit events or assess analytical solutions of specific boundary-value problems. They are, however, intractable for large scale simulations involving many dislocations. A computationally efficient variant of the unsteady diffusion problem was presented which assumes length scale separation, i.e., that concentration gradients are mainly determined at macroscopic scales, as would arise from boundary tractions. Under such circumstances, a "coarse-grained" formulation of the climb velocity is used whose robustness against atomistic calculations is documented (Clouet, 2011).

The salient features of the approach have been demonstrated for two-dimensional plane strain problems. First, creep emerged as a natural outcome of simulations. Second, some features of small-scale plasticity were discussed. Calculations have been reported for single crystalline strips subjected to simple tension under multislip conditions and for homologous temperatures in the range $0.4-0.85 T_{\mathrm{m}}$. Consistent with the recently reported 
(a)

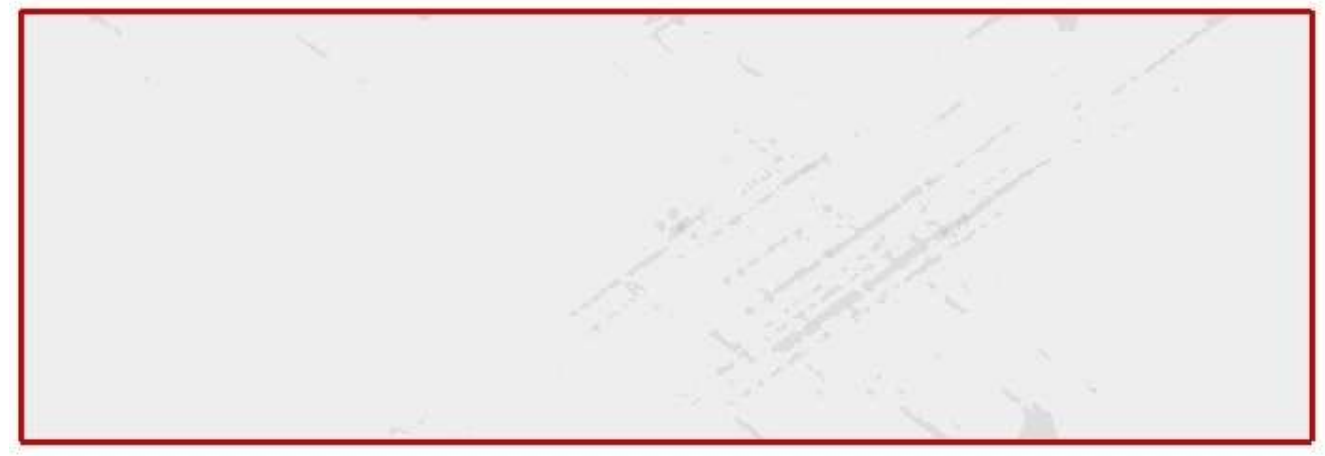

(b)

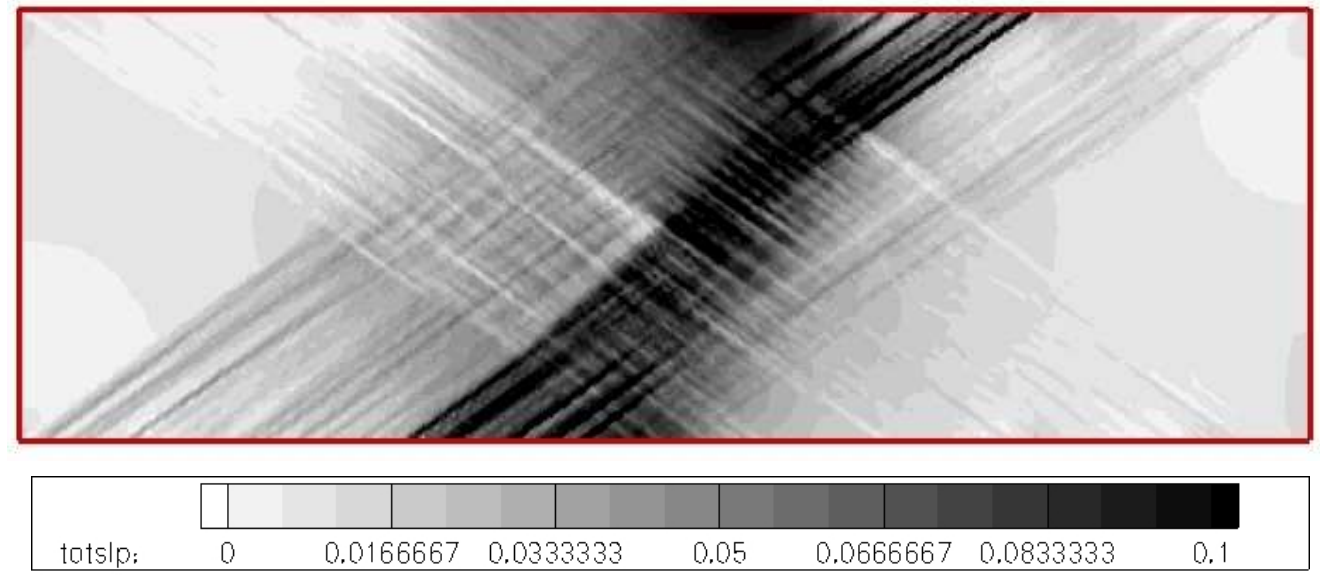

Figure 15: Contours of total slip $\Gamma$ in the specimen corresponding to the creep simulation of Fig. 14(b) in the time interval (a) $t=2500-3000$ seconds and (b) $t=3000-3500$ seconds.

findings of Keralavarma et al. (2012), the simulations exhibited a clear steady state regime with strain rates in reasonably good agreement with experimental data for polycrystals (Frost and Ashby, 1982). Examination of the deformation mechanism map in Fig. 1 shows that at $T=400 \mathrm{~K}\left(0.43 T_{\mathrm{m}}\right)$ the experimental range of the creep strain rates is about $10^{-8}$ $10^{-5} / \mathrm{s}$ for normalized shear stresses in the range $2 \times 10^{-4}-10^{-3}$. The predicted creep rates (Fig. 12) fall completely within this range. A noteworthy emergent behavior predicted by the calculations is the transition from diffusional creep (Herring-Nabarro) to dislocation creep (so-called power-law creep). The initial dislocation density was found to directly affect the magnitude of the creep strain rates, but not the creep exponents or the critical stress at which a crossover from diffusional to dislocation creep occurs (Fig. 12).

The simulations presented here contain idealizations in a number of respects. Any comparison between simulation results and experiments is restricted to qualitative aspects although some quantitative agreements are noted. There are some important threedimensional aspects of dislocation climb that could affect their subsequent glide mobility due to the inherent jogged character of the climbing dislocation. In the 2D simulations, 
the entire infinite edge dislocation climbs at once. In actuality, vacancy diffusion is not uniform along the dislocation line so that not all segments would climb at the same rate. Also, the presented simulations employed a minimal set of constitutive rules for the shortrange dislocation-dislocation interactions. As a result, creep occurred in a non-hardening material (Fig. 6a). While this simulation setup allowed a cleaner picture to be depicted with a constant background flow stress, there is interest in investigating the effect of hardening on creep evolution. A framework exists which will enable such interactions to be investigated in the future (Benzerga et al., 2004). In addition, the simulations currently ignore several aspects of the physics, such as pipe diffusion, which may play an important role in determining the creep rates at $T / T_{\mathrm{m}}$ in the lower range of the creep regime. Additional physics is also needed to model grain boundary mediated diffusion so that a distinction can be made between the two types of diffusional creep, Herring-Nabarro versus Coble creep. It is remarkable that despite the above idealizations, the proposed simulation framework provides physically sound predictions in addition to being computationally efficient.

There is great interest in creep-related dimensional stability in a host of miniature devices for electronic and medical applications as well as in nanostructured metals and composites, and at crack tips, particularly when the driving forces include chemical or thermal components. All such problems involve a size scale at which the combined glideclimb motion of large numbers of discrete dislocations plays a key role. At present, experimental data of thermally activated phenomena at such scales is scarce but certain features of creep in microscale specimens were captured by the simulations (Fig. 14). With further development of experimental nanomechanics at elevated temperatures the framework presented here is expected to enable fundamental understanding of deformation processes in small scale structures across a range of temperatures and competing multiple physics.

\section{Acknowledgements}

Support from the National Science Foundation under the Early Career program (grant CMMI-0748187) is gratefully acknowledged. Also, support from the Lawrence Livermore National Security, LLC under Master Task Agreements No. B599687 and B602391, LLNL under Contract DE-AC52-07NA27344 is acknowledged.

\section{Appendix}

Consider the continuity equation (15) in terms of the microscopic concentration field $c(\mathbf{x}, t)$ subject to boundary conditions on both $\partial V$ and $\partial C^{i}$. Define $\dot{C}$ as

$$
\dot{C}=\langle\dot{c}\rangle_{V_{g}}
$$


where $\langle\cdot\rangle_{V_{g}}$ stands for the volume average over some representative elementary volume or grain $V_{g}$. Using (15) one obtains

$$
\dot{C}=-\frac{1}{V_{g}} \int_{\hat{V}_{g}} \nabla \cdot \mathbf{j} d V-\frac{1}{V_{g}} \sum_{i \in V_{g}} \int_{\partial C^{i}} \mathbf{j} \cdot \mathbf{n} d S
$$

where $\hat{V}_{g}$ refers to excluding all dislocations cores from $V_{g}$. The net divergence of the macroscopic vacancy flux is then defined as

$$
\nabla \cdot \mathbf{J}=\frac{1}{V_{g}} \int_{\hat{V}_{g}} \nabla \cdot \mathbf{j} d V
$$

so that the second term on the right-hand side of (51), denoted $\dot{C}_{\text {src }}$, is clearly identified with vacancy production/emission within $V_{g}$. Considering closed dislocation loops none of which intersects the boundary of $V_{g}$, one gets

$$
\dot{C}_{\mathrm{src}}=-\frac{1}{V_{g}} \sum_{i \in V_{g}} \oint_{\mathcal{L}^{i}} \oint_{\Gamma^{i}} \mathbf{j} \cdot \mathbf{n} d s d l
$$

Using (17) one finally gets equation (29), which expresses the source/sink term as the net climb velocity of all the dislocations in $V_{g}$. A positive climb step of a dislocation requires the absorption of a single vacancy into the dislocation core while a negative climb step requires the emission of a single vacancy. Equation (30) is then obtained from (29) by considering that each edge dislocation climbs at one atomic site only. This obviously leads to a lower bound. On the other hand, an upper bound is obtained if one assumes that the whole edge dislocation climbs (one vacancy emitted/absorbed per lattice site along the dislocation line). The reality is somewhere in between depending on the lineal density of jogs.

\section{References}

Acharya, A., Bassani, J. L., 2000. Lattice incompatibility and a gradient theory of crystal plasticity. J. Mech. Phys. Solids 48, 1565-1595.

Arsenlis, A., Rhee, M., Hommes, G., Cook, R., Marian, J., 2012. A dislocation dynamics study of the transition from homogeneous to heterogeneous deformation in irradiated body-centered cubic iron. Acta Mater. 60, 3748-3757.

Asaro, R. J., Needleman, A., 1985. Overview no. 42 texture development and strain hardening in rate dependent polycrystals. Acta Metall. 33, 923-953.

Ashby, M., 1972. A first report on deformation-mechanism maps. Acta Metall. 20, 887897.

Ayas, C., Deshpande, V. S., Geers, M. G. D., 2012. Tensile response of passivated films with climb-assisted dislocation glide. Journal of the Mechanics and Physics of Solids 60, 1626-1643. 
Ayas, C., Van Dommelen, J. A. W., Deshpande, V. S., 2014. Climb-enabled discrete dislocation plasticity. J. Mech. Phys. Solids 62, 113-136.

Bakó, B., Clouet, E., Dupuy, L. M., Blétry, M., 2011. Dislocation dynamics simulations with climb: kinetics of dislocation loop coarsening controlled by bulk diffusion. Philosophical Magazine 91, 3173-3191.

Benzerga, A. A., 2008. An analysis of exhaustion hardening in micron-scale plasticity. Int. J. Plasticity 24, 1128-1157.

Benzerga, A. A., Bréchet, Y., Needleman, A., Van der Giessen, E., 2004. Incorporating three-dimensional mechanisms into two-dimensional dislocation dynamics. Model. Simul. Mater. Sci. Eng. 12, 159-196.

Benzerga, A. A., Bréchet, Y., Needleman, A., Van der Giessen, E., 2005. The stored energy of cold work: predictions from discrete dislocation plasticity. Acta Mater. 53, 4765-4779.

Bulatov, V., Abraham, F. F., Kubin, L., Devincre, B., Yip, S., 1998. Connecting atomistic and mesoscale simulations of crystal plasticity. Nature 391, 669-672.

Bullough, R., Newman, R. C., 1970. The kinetics of migration of point defects to dislocations. Reports on Progress in Physics 33, 101.

Caillard, D., Martin, J., 2003. Thermally Activated Mechanisms in Crystal Plasticity. Vol. 8. Elsevier Science, Amsterdam.

Castelnau, O., Duval, P., Lebensohn, R. A., Canova, G. R., 1996. Viscoplastic modeling of texture development in polycrystalline ice with a self-consistent approach: Comparison with bound estimates. J. of Geophys. Research-Solid Earth 101, 13851-13868.

Clouet, E., 2011. Predicting dislocation climb: Classical modeling versus atomistic simulations. Phys. Rev. B 84, 092106.

Coble, R. L., 1963. A model for boundary diffusion controlled creep in polycrystalline materials. J. App. Phys. 34 (6), 1679-1682.

Cocks, A., 1996. Variational principles, numerical schemes and bounding theorems for deformation by nabarro-herring creep. J. Mech. Phys. Solids 44, 1429-1452.

Cottrell, A. H., 1953. Dislocations and plastic flow in crystals. Oxford University Press, Oxford.

Cottrell, A. H., Jaswon, M. A., 1949. Distribution of solute atoms around a slow dislocation. Proceedings of the Royal Society of London. Series A. Mathematical and Physical Sciences 199, 104-114.

Davoudi, K. M., Nicola, L., Vlassak, J. J., 2012. Dislocation climb in two-dimensional discrete dislocation dynamics. J. App. Phys. 111, 103522. 
Dorn, J. E., 1955. Some fundamental experiments on high temperature creep. J. Mech. Phys. Solids 3, 85-116.

El-Azab, A., 2000. Statistical mechanics treatment of the evolution of dislocation distributions in single crystals. Phys. Rev. B 61, 11956-11966.

Fleck, N. A., Hutchinson, J. W., 1997. Strain gradient plasticity. Adv. Appl. Mech. 33, 295-361.

Freund, P., Heinloth, K., 2002. Numerical Data and Functional Relationships in Science and Technology: New Series. Springer, New York.

Frost, H. J., Ashby, M. F., 1982. Deformation-Mechanism Maps: The Plasticity and Creep of Metals and Ceramics. Pergamon Press, Oxford.

Gao, Y., Cocks, A., 2009. Thermodynamic variational approach for climb of an edge dislocation. Acta Mech. Solida Sin. 22, 426-435.

Gao, Y., Zhuang, Z., Liu, Z., You, X., Zhao, X., Zhang, Z., 2010. Investigations of pipediffusion-based dislocation climb by discrete dislocation dynamics. Int. J. Plasticity 27, $1055-1071$.

Garikipati, K., Falk, M., Bouville, M., Puchala, B., Narayanan, H., 2006. The continuum elastic and atomistic viewpoints on the formation volume and strain energy of a point defect. J. Mech. Phys. Solids 54, 1929-1951.

Geers, M., Cottura, M., Appolaire, B., Busso, E., Forest, S., Villani, A., 2014. Coupled glide-climb diffusion-enhanced crystal plasticity. J. Mech. Phys. Solids 70, 136-153.

Geslin, P., Appolaire, B., Finel, A., 2014. A phase field model for dislocation climb. Appl. Phys. Lett. 104, 011903.

Ghoniem, N. M., Tong, S. H., Sun, L. Z., 2000. Parametric dislocation dynamics: A thermodynamics-based approach to investigations of mesoscopic plastic deformation. Phys. Rev. B 61, 913-927.

Greer, J. R., Oliver, W. C., Nix, W. D., 2005. Size dependence of mechanical properties of gold at the micron scale in the absence of strain gradients. Acta Mater. 53, 1821-1830.

Gurtin, M. E., 2002. A gradient theory of single-crystal viscoplasticity that accounts for the geometrically necessary dislocations. J. Mech. Phys. Solids 50, 5-32.

Harrison, E., Wilkes, P., 1972. The nature of the vacancy in Aluminum. Acta Metall. 20, $31-36$.

Hartmaier, A., Buehler, M. J., Gao, H. J., 2005. Two-dimensional discrete dislocation models of deformation in polycrystalline thin metal films on substrates. Mater. Sci. Eng. A-Struct. 400, 260-263. 
Herring, C., 1950. Diffusional viscosity of a polycrystalline solid. J. App. Phys. 21, 437445.

Hindmarsh, A., et al., 1983. ODEPACK, a systematized collection of ODE solvers. Scientific Computing 1, 55-64.

Hirth, J. P., Lothe, J., 1968. Theory of Dislocations. Wiley, New York.

Hochrainer, T., Sandfeld, S., Zaiser, M., Gumbsch, P., 2014. Continuum dislocation dynamics: towards a physical theory of crystal plasticity. J. Mech. Phys. Solids 63, 167178.

Ispánovity, P. D., Laurson, L., Zaiser, M., Groma, I., Zapperi, S., Alava, M. J., 2014. Avalanches in $2 \mathrm{~d}$ dislocation systems: Plastic yielding is not depinning. Phys. Rev. Lett. 112, 235501.

Keralavarma, S. M., 2011. A Contribution to the Modeling of Metal Plasticity and Fracture: From Continuum to Discrete Descriptions. Ph.D. thesis, Texas A\&M University, USA.

Keralavarma, S. M., Cagin, T., Arsenlis, A., Benzerga, A. A., 2012. Power-Law Creep From Discrete Dislocation Dynamics. Phys. Rev. Lett. 109, 265504.

Li, Y., Hu, S., Henager Jr, C. H., Deng, H., Gao, F., Sun, X., Khaleel, M. A., 2012. Computer simulations of interstitial loop growth kinetics in irradiated bcc fe. J. Nuc. Mater. 427, 259-267.

McDowell, D. L., 2008. Viscoplasticity of heterogeneous metallic materials. Mater Sci Eng:R 62, 67-123.

Meyers, M. A., Mishra, A., Benson, D. J., 2006. Mechanical properties of nanocrystalline materials. Prog. Mater. Sci. 51, 427-556.

Mordehai, D., Clouet, E., Fivel, M., Verdier, M., 2008. Introducing dislocation climb by bulk diffusion in discrete dislocation dynamics. Phil. Mag. 88, 899-926.

Nabarro, F. R. N., Basinski, Z. S., Holt, D. B., 1964. The Plasticity of Pure Single Crystals. Adv. Phys. 13, 193-323.

Ng, K., Ngan, A., 2007. Creep of micron-sized aluminium columns. Phil. Mag. Letters 87, 967-977.

Po, G., Ghoniem, N., 2014. A variational formulation of constrained dislocation dynamics coupled with heat and vacancy diffusion. J. Mech. Phys. Solids 66, 103-116.

Raabe, D., 1998. On the consideration of climb in discrete dislocation dynamics. Phil. Mag. A 77, 751-759.

Schilling, W., 1978. Self-interstitial atoms in metals. J. Nucl. Mater. 69, 465-489. 
Shenoy, V. B., Miller, R., Tadmor, E. b., Rodney, D., Phillips, R., Ortiz, M., 1999. An adaptive finite element approach to atomic-scale mechanics-the quasicontinuum method. J. Mech. Phys. Solids 47, 611-642.

Shilkrot, L. E., Miller, R. E., Curtin, W. A., 2004. Multiscale plasticity modeling: coupled atomistics and discrete dislocation mechanics. J. Mech. Phys. Solids 52, 755-787.

Shishvan, S. S., Van der Giessen, E., 2010. Distribution of dislocation source length and the size dependent yield strength in freestanding thin films. J. Mech. Phys. Solids 58, 678-695.

Uchic, M. D., Dimiduk, D. M., Florando, J. N., Nix, W. D., 2004. Sample dimensions influence strength and crystal plasticity. Science 305, 986-989.

Van der Giessen, E., Needleman, A., 1995. Discrete dislocation plasticity: a simple planar model. Model. Simul. Mater. Sci. Eng. 3, 689-735.

Vattré, A., Devincre, B., Feyel, F., Gatti, R., Groh, S., Jamond, O., Roos, A., 2014. Modelling crystal plasticity by $3 \mathrm{~d}$ dislocation dynamics and the finite element method: The discrete-continuous model revisited. J. Mech. Phys. Solids 63, 491-505.

Weertman, J., 1957. Steady-state creep through dislocation climb. J. App. Phys. 28, 362364.

Xiang, Y., Srolovitz, D. J., Cheng, L.-T., Weinan, E., 2004. Level set simulations of dislocation-particle bypass mechanisms. Acta Mater. 52, 1745-1760.

Zbib, H., Rhee, M., Hirth, J. P., 1998. On plastic deformation and the dynamics of 3D dislocations. Int. J. Mech. Sci. 40, 113-127. 